\title{
The Virtual Brain: a simulator of primate brain network dynamics
}

\section{Paula Sanz Leon ${ }^{1 *}$, Stuart A. Knock ${ }^{2}$, M. Marmaduke Woodman ${ }^{1}$, Lia Domide ${ }^{3}$, Jochen Mersmann ${ }^{4}$, Anthony R. Mclntosh ${ }^{5}$ and Viktor Jirsa ${ }^{1 *}$}

1 Institut de Neurosciences des Systèmes, Aix Marseille Université, Marseille, France

2 Department of Neurology, BrainModes Group, Charité University of Medicine, Berlin, Germany

${ }^{3}$ Codemart, Cluj-Napoca, Romania

${ }^{4}$ CodeBox $\mathrm{GmbH}$, Stuttgart, Germany

${ }^{5}$ Rotman Research Institute at Baycrest, Toronto, ON, Canada

\section{Edited by:}

Daniele Marinazzo, University of Gent, Belgium

\section{Reviewed by:}

Ingo Bojak, University of Reading, UK

Hans Ekkehard Plesser, Norwegian University of Life Sciences, Norway Laurent U. Perrinet, Centre National de la Recherche Scientifique, France

\section{*Correspondence:}

Paula Sanz Leon and Viktor Jirsa, Institut de Neurosciences des

Systèmes, Aix Marseille

Université, 27, Bd. Jean Moulin,

13005 Marseille, France

e-mail: paula.sanz-leon@univ-amu.fr;

viktor.jirsa@univ-amu.fr
We present The Virtual Brain (TVB), a neuroinformatics platform for full brain network simulations using biologically realistic connectivity. This simulation environment enables the model-based inference of neurophysiological mechanisms across different brain scales that underlie the generation of macroscopic neuroimaging signals including functional MRI (fMRI), EEG and MEG. Researchers from different backgrounds can benefit from an integrative software platform including a supporting framework for data management (generation, organization, storage, integration and sharing) and a simulation core written in Python. TVB allows the reproduction and evaluation of personalized configurations of the brain by using individual subject data. This personalization facilitates an exploration of the consequences of pathological changes in the system, permitting to investigate potential ways to counteract such unfavorable processes. The architecture of TVB supports interaction with MATLAB packages, for example, the well known Brain Connectivity Toolbox. TVB can be used in a client-server configuration, such that it can be remotely accessed through the Internet thanks to its web-based HTML5, JS, and WebGL graphical user interface. TVB is also accessible as a standalone cross-platform Python library and application, and users can interact with the scientific core through the scripting interface IDLE, enabling easy modeling, development and debugging of the scientific kernel. This second interface makes TVB extensible by combining it with other libraries and modules developed by the Python scientific community. In this article, we describe the theoretical background and foundations that led to the development of TVB, the architecture and features of its major software components as well as potential neuroscience applications.

Keywords: connectome, neural masses, time delays, full-brain network model, virtual brain, large-scale simulation, web platform, python

\section{INTRODUCTION}

Brain function is thought to emerge from the interaction of large numbers of neurons, under the spatial and temporal constraints of brain structure and cognitive demands. Contemporary network simulations mainly focus on the microscopic and mesoscopic level (neural networks and neural masses representing a particular cortical region), adding detailed biophysical information at these levels of description while too often losing perspective on the global dynamics of the brain. On the other hand, the degree of assessment of global cortical dynamics, across imaging modalities, in human patients and research subjects has increased significantly in the last few decades. In particular, cognitive and clinical neuroscience employs imaging methods of macroscopic brain activity such as intracerebral measurements, stereotactic Encephalography (sEEG) (von Ellenrieder et al., 2012), Electroencephalography (EEG) (Nunez and Srinivasan, 1981; Nunez, 1995; Niedermeyer and Lopes Da Silva, 2005), Magnetoencephalography (MEG) (Hämäläinen,
1992; Hämäläinen et al., 1993; Mosher et al., 1999), and functional Magnetic Resonance Imaging (fMRI) (Ogawa et al., 1993, 1998; Logothetis et al., 2001) to assess brain dynamics and evaluate diagnostic and therapeutic strategies. Hence, there is a strong motivation to develop an efficient, flexible, neuroinformatics platform on this macroscopic level of brain organization for reproducing and probing the broad repertoire of brain dynamics, enabling quick data analysis and visualization of the results.

The Virtual Brain (TVB) is our response to this need. On the one hand, it provides a general infrastructure to support multiple users handling various kinds of empirical and simulated data, as well as tools for visualizing and analyzing that data, either via internal mechanisms or by interacting with other computational systems such as MATLAB. At the same time it provides a simulation toolkit to support a top-down modeling approach to whole brain dynamics, where the underlying network is defined by its structural large-scale connectivity and mesoscopic models that govern the nodes' intrinsic dynamics. The interaction with the 
dynamics of all other network nodes happens through the connectivity matrix via specific connection weights and time delays, where the latter make a significant contribution to the biological realism of the temporal structure of dynamics.

Historically, Jirsa et al. (2002) first demonstrated neural field modeling on a spherical brain hemisphere employing EEG and MEG forward solutions to obtain simulation imaging signals. In this work, homogeneous (translationally invariant) connectivity was implemented along the lines of Jirsa and Haken (1996, 1997); Bojak and Liley (2010) yielding a neural field equation, which has its roots in classic works (Wilson and Cowan, 1972, 1973; Nunez, 1974; Amari, 1975, 1977). At that time more detailed large-scale connectivity of the full primate brain was unavailable, hence the homogeneous connectivity scaled up to the full brain was chosen as a first approximation (Nunez, 1974). The approach proved successful for the study of certain phenomena as observed in large-scale brain systems including spontaneous activity (Wright and Liley, 1995; Robinson et al., 2001, 2003; Breakspear et al., 2003; Rowe et al., 2004; Freyer et al., 2011), evoked potentials (Rennie et al., 1999, 2002), anesthesia (Liley and Bojak, 2005), epilepsy (Breakspear et al., 2006), sensorimotor coordination (Jirsa and Haken, 1996, 1997), and more recently, plasticity (Robinson, 2011) [see Deco et al. (2008) and Jirsa (2004) for a review].

Careful review of this literature though shows that these models mostly emphasize the temporal domain of brain organization, but leave the spatiotemporal organization untouched. This may be understood by the fact that the symmetry of the connectivity imposes constraints upon the range of the observable dynamics. This was pointed out early by Jirsa et al. (2002) and a suggestion was made to integrate biologically realistic DTI based connectivity into full brain modeling efforts. Large scale brain dynamics are basically expected to reflect the underlying anatomical connectivity between brain areas (Bullmore and Sporns, 2009; Deco et al., 2011), even though structural connectivity is not the only constraint, but the transmission delays play an essential role in shaping the brain network dynamics also (Jirsa and Kelso, 2000; Ghosh et al., 2008; Knock et al., 2009; Jirsa et al., 2010). Recent studies (Pinotsis et al., 2012) have systematically investigated the degree to which homogeneous approximations may serve to understand realistic connection topologies and have concluded that homogeneous approximations are more appropriate for mesoscopic descriptions of brain activity, but less well suited to address full brain network dynamics. All this underscores the need to incorporate realistic connectivity into large scale brain network models. Thus the simulation side of TVB has evolved out of a research program seeking to identify and reproduce realistic whole brain network dynamics, on the basis of empirical connectivity and neural field models (Jirsa and Stefanescu, 2010; Deco et al., 2011).

\subsection{MODELING}

In line with these previous studies, TVB incorporates a biologically realistic, large-scale connectivity of brain regions in the primate brain. Connectivity is mediated by long-range neural fiber tracts as identified by tractography based methods (Hagmann et al., 2008; Honey et al., 2009; Bastiani et al., 2012), or obtained from CoCoMac neuroinformatics database (Kötter, 2004; Kötter and Wanke, 2005; Bakker et al., 2012). In TVB, the tract-lengths matrix of the demonstration connectivity dataset is symmetric due to the fiber detection techniques used to extract the information being insensitive to directionality. On the other hand, the weights matrix is asymmetric as it makes use of directional information contained in the tracer studies of the CoCoMac database. Such details are specific to the connectivity demonstration dataset included in the distribution packages of TVB. The symmetry (or lack thereof) is neither a modeling constraint nor an imposed restriction on the weights and tract-length matrices. The general implementation for weights and tract lengths are full nodes $\times$ nodes matrices without any symmetry restrictions.

Two types of structural connectivity are distinguished in TVB, that is long- and short-range connectivity, given by the connectivity matrix and the folded cortical surface, respectively. The connectivity matrix defines the connection strengths and time delays via finite signal transmission speed between two regions of the brain. The cortical surface allows a more detailed spatial sampling resulting in a spatially continuous approximation of the neural activity as in neural field modeling (Deco et al., 2008; Coombes, 2010; Bressloff, 2012). When using neural mass models, building the network upon the surface allows for the application of arbitrary local connectivity kernels which represent short-range intra-cortical connections. Additionally, networks themselves can be defined at two distinct spatial scales yielding two types of simulations (or brain network models): surface-based and region-based. In the former case, cortical and sub-cortical areas are shaped more realistically, each vertex of the surface is considered a node and is modeled by a neural population model; several nodes belong to a specific brain region, and the edges of the network have a distance of the order of a few millimeters. The influence of delayed activity coming from other brain regions is added to the model via the long-range connectivity. In the latter case of nodes only per region, the connectome itself is used as a coarser representation of the brain network model. The networks comprise discrete nodes, each of which models the neural population activity of a brain region and the edges represent the long-range connectivity (interregional fibers) on the order of a few centimeters. Consequently, in surface-based simulations both types of connectivity, short- and long-range, coexist whereas in region-based simulations one level of geometry is lost: the short-range connectivity.

Neural field models have been developed over many years for their ability to capture the collective dynamics of relatively large areas of the brain in both analytically and computationally tractable forms (Beurle, 1956; Wilson and Cowan, 1972, 1973; Nunez, 1974; Amari, 1975, 1977; Wright and Liley, 1995; Jirsa and Haken, 1996, 1997; Robinson et al., 1997; Jirsa et al., 2002; Atay and Hutt, 2006; Bojak and Liley, 2010). Effectively neural field equations are tissue level models that describe the spatiotemporal evolution of coarse grained variables such as synaptic voltage or firing rate activity in populations of neurons. Some of these models include explicit spatial terms while others are formulated without an explicit spatial component leaving open the possibility to apply effectively arbitrary local connectivity kernels. The lumped representation of the dynamics of a set of similar neurons 
via a common variable (e.g., mean firing rate and mean postsynaptic potential) is known as neural mass modeling (Freeman, 1975, 1992; Lopes da Silva et al., 1974). Neural mass models accounting for parameter dispersion in the neuronal parameters include Assisi et al., 2005; Stefanescu and Jirsa, 2008, 2011; Jirsa and Stefanescu, 2010. Networks of neural masses, without an explicit spatial component within the mass but with the possibility to apply local connectivity kernels (e.g., Gaussian or Laplacian functions) between masses, can be used to approximate neural field models. Both neural field and neural mass modeling approaches embody the concept from statistical physics that macroscopic physical systems obey laws that are independent of the details of the microscopic constituents of which they are built (Haken, 1983). These and related ideas have been exploited in neurosciences (Kelso, 1995; Buzsaki, 2006).

In TVB, our main interest lies in using the mesoscopic laws governing the behavior of neural populations and uncovering the laws driving the processes on the macroscopic brain network scale. The biophysical mechanisms available to microscopic single neuron approaches are absorbed in the mean field parameters on the mesoscopic scale and are not available for exploration other than through variation of the mean field parameters themselves. As a consequence, TVB represents a neuroinformatics tool that is designed to aid in the exploration of large-scale network mechanisms of brain functioning [see Ritter et al. (2013) for an example of modeling with TVB].

Furthermore, TVB's approach to multi-modal neuroimaging integration in conjunction with neural field modeling shares common features with the work of Bojak et al. $(2010,2011)$ and Babajani-Feremi and Soltanian-Zadeh (2010). The crucial difference is that the structure upon which TVB has been designed represents a generalized large-scale "computational neural model" of the whole brain. The components of this large-scale model have been separated as cleanly as possible, and a specific structure has been defined for the individual components. This generic structure is intended to serve the purpose of restricting the form of models enough to make direct comparison straight forward while still permitting a sufficiently large class of models to be expressed. Likewise, the paradigms presented during the last few years in this line of research (Sotero et al., 2007; Sotero and Trujillo-Barreto, 2008) could potentially be reproduced, tested and compared in TVB. The mathematics underlying our model-based approach have been partially described in various original articles (Deco et al., 2011; Deco and Jirsa, 2012) and will be reviewed in more detail in future articles.

\subsection{INFORMATICS}

From an informatics perspective, a large-scale simulation project requires a well defined yet flexible workflow, i.e., adaptable according to the users profiles. A typical workflow in TVB involves managing project information, uploading data, setting up simulation parameters (model, integration scheme, output modality), launching simulations (in parallel if needed), analyzing and visualizing, and finally storing results and sharing output data.

The web interface allows users without programming knowledge to access TVB to perform customized simulations (e.g., clinicians could use their patient's data obtained from DTI studies). In addition, it enables them to gain a deeper understanding of the theoretical approaches behind the scenes. On the other hand, theoreticians can design their own models and get an idea of their biophysical realism, their potential physiological applications and implications. As both kinds of users may work within the same framework, the interplay of theory and experiment or application is accelerated. Additionally, users with stronger programming skills benefit from all the advantages provided by the Python programming language: easy-to-learn, easy-to-use, scriptable and with a large choice of scientific modules (Oliphant, 2006).

TVB has been principally built in the Python programming language due to its unique combination of flexibility, existing libraries and the ease with which code can be written, documented, and maintained by non-programmers. The simulation core, originally developed in MATLAB, was ported to Python given its current significance in the numerical computing and neuroscience community and its already proven efficiency for implementing modeling tools (Spacek et al., 2008).

Simulations benefit from vectorized numerical computations with NumPy arrays and are enhanced by the use of the numexpr package. Although this allows rather efficient single simulations, the desire to systematically explore the parameter spaces of the neural dynamic models, and to compare many connectivity matrices, has lead to the implementation of code generation mechanisms for the majority of the simulator core-producing $\mathrm{C}$ code for both native CPU and also graphics processing units (GPU), leading to a significant speed up of parameter sweeps and parallel simulations ( $5 \mathrm{x}$ from Python to $\mathrm{C}, 40 \mathrm{x}$ from $\mathrm{C}$ to GPU). Such graphics units have become popular in scientific computing for their relatively low price and high computing power. Going forward, the GPU implementation of TVB will require testing and optimization before placing it in the hands of users.

This article intends to give a comprehensive but nonexhaustive description of TVB, from both technical and scientific points of view. It will describe the framework's architecture, the simulation core, and the user interfaces. It will also provide two examples, using specific features of the simulator, extracted from the demo scripts which are currently available in TVB's distribution packages.

\section{TVB ARCHITECTURE}

The architectural model of the system has two main components: the scientific computing core and the supporting framework with its graphical user interface. Both software components communicate through an interface represented by TVB-Datatypes, which are described in section 2.2. In Figure 1 TVB's architectural details are illustrated and explained in more depth.

General aspects: TVB is designed for three main deployment configurations, according to the available hardware resources: (1) Stand Alone; (2) Client-Server or, (3) Cluster. In the first, a local workstation is assumed to have certain display, computing power and storage capacity resources. In the second, an instance of TVB is running on a server connected through a network link to client units, and thus accessible to a certain number of users. 


\section{TVB - architectural view}

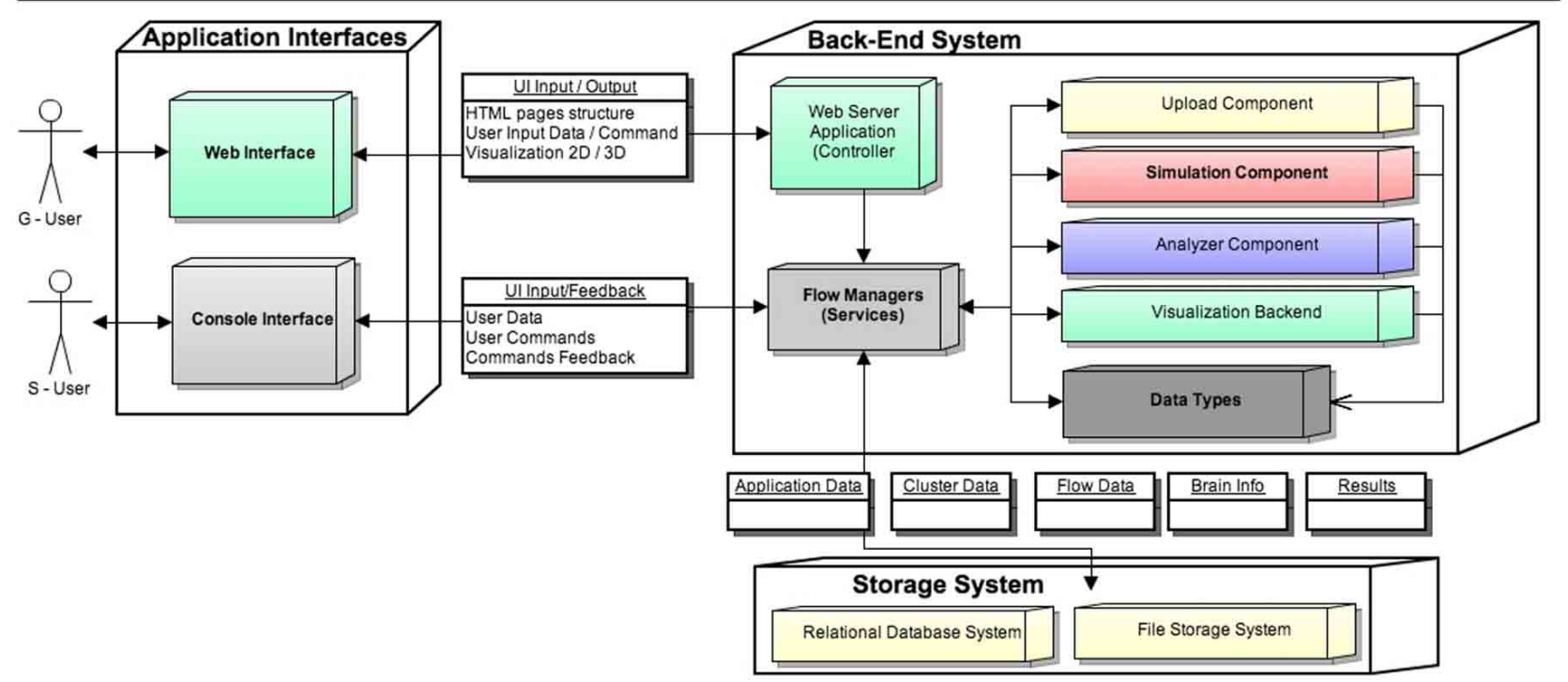

FIGURE 1| The Virtual Brain Architecture: TVB provides two independent interfaces depending on the interaction with users. Blocks in the back-end are transparently used by different top application layers. TVB-Datatypes, are the common language between different components (analyzers, visualizers, simulator, uploaders). They represent "active data" in the sense that, when TVB is configured with a database, data contained in TVB-Datatypes instances are automatically persistent. Currently the console interface works without the storage layer, keeping the results just in memory. S-Users need to manually handle data import and export operations.
In this deployment model, simulations use the back-end server's computing power while visualization tasks use resources from the client machine. The third is similar to the client-server configuration, but with the additional advantage of parallelization support in the back-end. The cluster itself needs to be configured separately of TVB.

Based on the usage scenarios and user's level of programming knowledge, two user profiles are represented: a graphical user (G-user) and scripting user (S-user). We therefore provide the corresponding main interfaces based on this classification: a graphical user interface (web) and a scripting interface (IDLE). S-users and G-users have different levels of control over different parts of the system. The profile of S-users is thought to be that of scientific developers, that is, researchers who can elaborate complex modeling scenarios, add their own models or directly modify the source code to extend the scientific core of TVB, mostly working with the scientific modules. They do, nevertheless, have the possibility to enable the database system. In contrast, G-users are relatively more constrained to the features available in the stable releases of TVB, since their profile corresponds more to that of researchers without a strong background in computational modeling. The distinction between these two profiles is mainly a categorization due to the design architecture of TVB. For instance, we could also think of other type of users who want to work with TVB's GUI and are comfortable with programming, and therefore they could potentially make modifications in the code and then see the effect of those when launching the application in a web browser.

The development of TVB is managed under Agile techniques. In accord therewith, each task is considered as done, after completing a validation procedure that includes: adding a corresponding automated unit-test, labeling the task as finished from the team member assigned to implement the task and further tagging as closed from a team member responsible for the module, which means a second level of testing. Before releasing stable packages, there is a period for manual testing, that is, a small group of selected users from different institutions check the main features and functionalities through both interfaces. The navigation and workflows scenarios through the webbased interface are evaluated by means of automated integration tests for web-applications running with Selenium (http://docs. seleniumhq.org/) and Apache-JMeter (http://jmeter.apache.org/) on top of a browser engine. Special effort is being made to provide good code-coverage, including regression tests. Accordingly, the simulation engine of TVB has automated unit-tests, to guarantee the proper and coordinated functioning of all the modules, and simple programs (demonstration scripts), that permit qualitative evaluation of the scientific correctness of results.

The development version of TVB is currently hosted on a private cluster, where we use the version control system svn (subversion). Additionally, as any large collaborative open-source project, it is also available in a public repository, using the distributed version control system git (Chacon, 2009) to make accessible the scientific core and to gather, manage and integrate contributions from the community. The distribution packages for TVB come with an extensive documentation, including: a User Guide, explaining how to install TVB, set up models and run them; Tutorials, Use Cases and Script Demos, guiding users to achieve predefined simulation scenarios; and a Developer Guide and API reference. Table 1 provides the links 


\section{Table 1 | TVB links.}

TVB official website

Distribution packages

Public repository

User group http://www.thevirtualbrain.org/register https://github.com/the-virtual-brain https://groups.google.com/group/tvb-users/ http://www.thevirtualbrain.org

to: the official TVB website, where distribution packages for Linux and Mac OS (32 and 64 bits) and Windows (32 bits) are available for download; the active users group of TVB hosted in Google Groups, where users can ask questions, report issues and suggest improvements or new features; and the public repository, where the source code of both the framework and scientific library (which contains the simulation engine) are available.

Installation and System Requirements: When using the web interface, users are recommended to have a high definition monitor (at least $1600 \times 1000$ pixels), a WebGL and WebSockets compatible browser (latest versions of Mozilla Firefox, Apple Safari or Google Chrome), and a WebGL-compatible graphics card, that supports OpenGL version 2.0 or higher (Shreiner et al., 2005).

Regarding memory and storage capacity, for a stand alone installation a minimum of $8 \mathrm{~GB}$ of RAM is recommended. For multi-users environments $5 \mathrm{~GB}$ of space per user is suggested. This is a storage quota specified by an administrator to manage the maximum hard disk space per user. As for computing power one CPU core is needed for a simulation with a small number of nodes, while simulations with a large number of nodes, such as surface simulations, can make use of a few cores if they are available. When the number of launched simulations is larger than the number of available cores, a serialization is recommended (a serialization mechanism is provided by the supporting framework through the web user interface by specifying the maximum of simultaneous jobs allowed). In order to use the Brain Connectivity Toolbox (Rubinov and Sporns, 2010), MATLAB or Octave should be installed, activated and accessible for the current user.

\subsection{TVB FRAMEWORK}

The supporting framework provides a database back-end, workflow management and a number of features to support collaborative work. The latter feature permits TVB to be setup as a multi-user application. In this configuration, a login system enables users to access their personal sessions; by default their projects and data are private, but they can be shared with other users. The graphical user interface (GUI) is web based, making use of HTML 5, WebGL, CSS3 and Java Script (Bostock et al., 2011) tools to provide an intuitive and responsive interface that can be locally and remotely accessed.

\subsubsection{Web-based GUI}

TVB provides a web-based interactive framework to generate, manipulate and visualize connectivity and network dynamics. Additionally, TVB comprises a set of classic time-series analysis tools, structural and functional connectivity analysis tools, as well as parameter exploration facilities which can launch simulations in parallel on a cluster or on multiple compute cores of a server. The GUI of TVB has six main working areas: USER, PROJECT, SIMULATOR, ANALYZE, STIMULUS, and CONNECTIVITY. In USER, the users manage their accounts and TVB settings. In PROJECT, individual projects are managed and navigation tools are provided to explore their structure as well as the data associated with them. A sub-menu within this area provides a dashboard with a list of all the operations along with their current status (running, error, finished), owner, wall-time and associated data, among other information. In SIMULATOR the large-scale network model is set up and simulations launched, additional viewers for structural and functional data are offered in $2 \mathrm{D}$ and $3 \mathrm{D}$, as well as other displays to visualize the results of a simulation. A history of simulations is also available in this area. In ANALYZE time-series and network analysis methods are provided. In STIMULUS, users can interactively create stimulation patterns. Finally, in CONNECTIVITY, users are given a responsive interface to edit the connectivity matrices assisted by interactive visualization tools. Figure 2 depicts the different working areas, as well as a number of their sub-menus, available through the web UI. A selection of screenshots illustrating the interface in a web browser is given in Figure 3.

\subsubsection{Data management and exchange}

One of the goals of TVB is to allow researchers from different backgrounds and with different programming skills to have quick access to their simulated data. Data from TVB can be exchanged with other instances of TVB (copies installed on different computers) or with other applications in the neuroscientific community, e.g., MATLAB, Octave, The Connectome ToolKit (Gerhard et al., 2011).

Export: A project created within TVB can be entirely exported to a .zip file. Besides storing all the data generated within a particular project in binary files, additional XML files are created to provide a structured storage of metadata, especially with regard to the steps taken to set up a simulation, configuration parameters for specific operations, time-stamps and user account information. This mechanism produces a summary of the procedures carried on by researchers within a project which is used for sharing data across instances of TVB. The second export mechanism allows the export of individual data objects. The data format used in TVB is based on the HDF5 format (The HDF Group, 2010) because it presents a number of advantages over other formats: (1) huge pieces of data can be stored in a condensed form; (2) it allows grouping of data in a tree structure; (3) it allows metadata assignment at every level; and (4) it is a widely used format, accessible in several programming languages and applications. Additionally, each object in TVB has a global unique identifier (GUID) which makes it easy to identify an object across systems, avoiding naming conflicts among files containing objects of the same type.

Import: A set of mechanisms ("uploaders") is provided in TVB to import data into the framework, including neuroimaging data generated independently by other applications. The following formats are supported: NIFTI-1 (volumetric time- series), GIFTI 


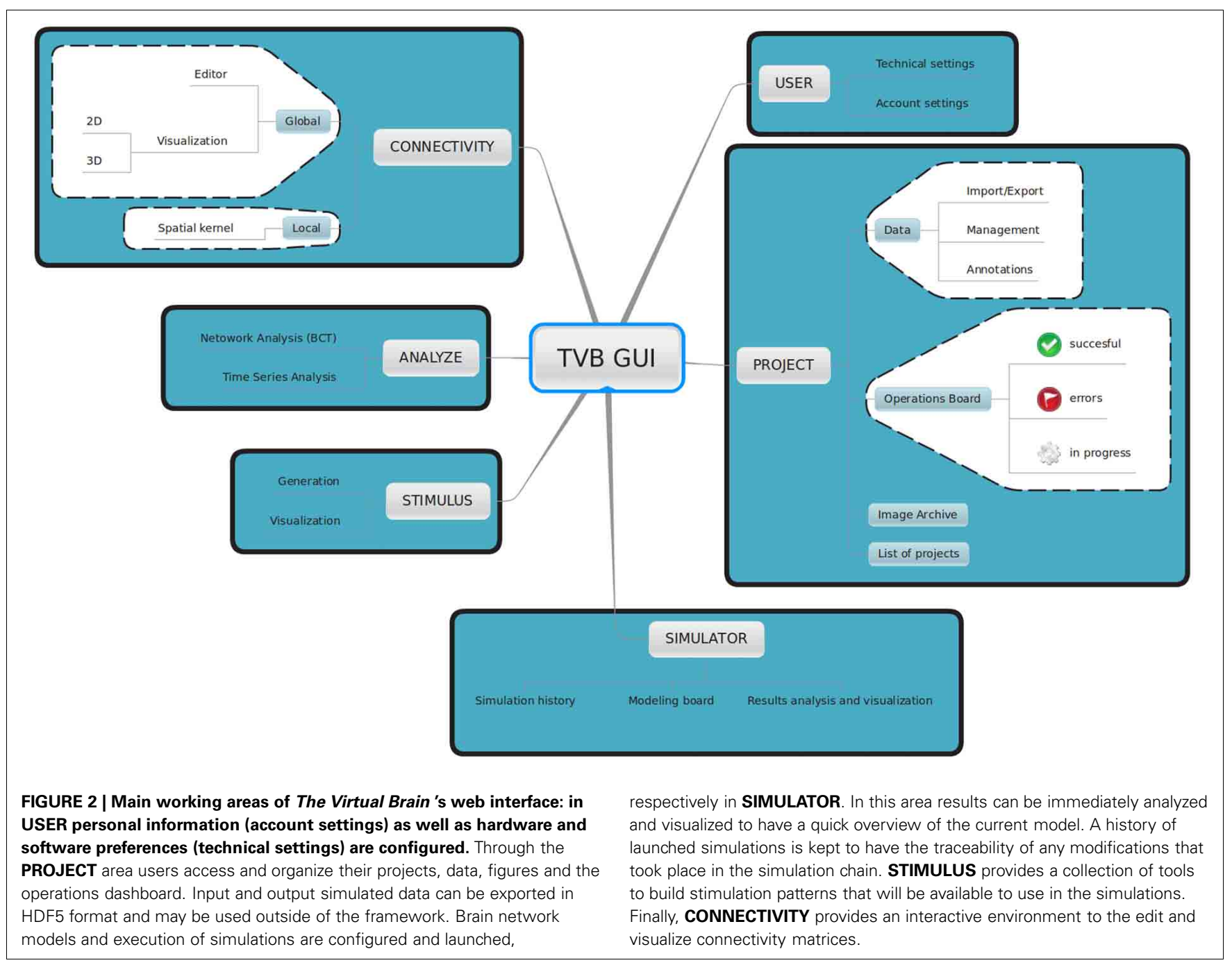

(surfaces) and CFF (connectome file). General compression formats, such as ZIP and BZIP2 are also supported for certain data import routines that expect a set of ASCII text files compressed in an archive. Hence the use of general compression formats means that preparing datasets for TVB is as simple as generating an archive with the correct ASCII files, in contrast to some of the other neuroscientific data formats found elsewhere. For instance, a Connectivity dataset (connectome) may be uploaded as a zip folder containing the following collection of files: (1) areas.txt, (2) average_orientations.txt, (3) info.txt, (4) positions.txt, (5) tract_lengths.txt, and (6) weigths.txt. More conventions and guidelines to use each uploader routine can be found in the User Guide of TVB's documentation.

\subsubsection{File storage}

The storage system is a tree of folders and files. The actual location on disk is configurable by the user, but the default is a folder called "TVB" in the user's home folder. There is a sub-folder for each Project in which an XML file containing details about the project itself is stored. Then for each operation, one folder per operation is created containing a set of .h5 files generated during that particular operation, and one XML file describing the operation itself. The XML contains tags like creation date, operation status (e.g., Finished, Error), algorithm reference, operation GUID, and most importantly input parameters dictionary. Sufficiently detailed information is stored in the file system to be able to export data from one instance of TVB and to then import it into another instance, correctly recreating projects, including all operations and their results. Even though the amount of data generated per operation varies greatly, since it depends strongly on the Monitors used and parameters of the simulation, some rough estimates are given below:

- A $1000 \mathrm{~ms}$ long, region-based simulation with all the default parameters requires approximatively $1 \mathrm{MB}$ of disk space.

- A $10 \mathrm{~ms}$ long, surface-based simulation, using a precalculated sparse matrix to describe the local connectivity kernel and all the default parameters, requires about $280 \mathrm{MB}$.

Users can manually remove unused data using the corresponding controls in TVB's GUI. In this case, all files related to these 


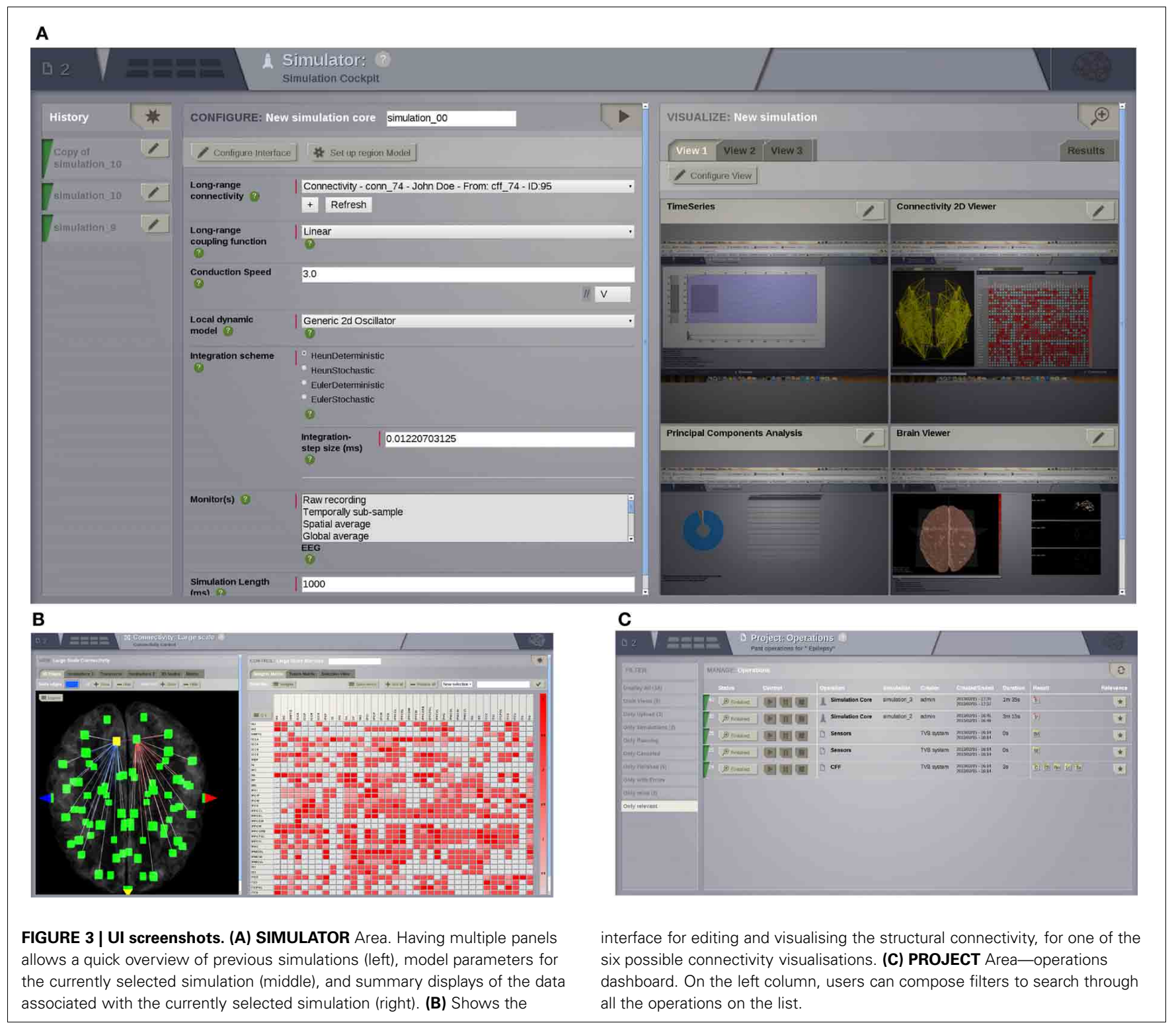

data are also deleted, freeing disk space. The amount of physical storage space available to TVB can be configured in the USER $\rightarrow$ Settings working area of the GUI-this is, of course, limited by the amount of free space available on the users hard drives.

\subsubsection{Database management system}

Internally, TVB framework uses a relational database (DB), for ordering and linking entities and as an indexing facility to quickly look up data. At install time, users can choose between SQLite (a file based database and one of the most used embedded DB systems) and PostgreSQL (a powerful, widely spread, open-source object-relational DB system which requires a separate installation by users) as the DB engine. In the database, only references to the entities are stored, with the actual operation results always being stored in files, due to size. A relational database was chosen as it provides speed when filtering entities and navigating entity relationship trees.

\subsection{TVB DATATYPES}

In the architecture of TVB, a middleware layer represented by TVB-Datatypes allows the handling and flow of data between the scientific kernel and the supporting framework. TVB-Datatypes are annotated data structures which contain one or more data attributes and associated descriptive information, as well as methods for operating on the data they contain. The definition of a Datatype is achieved using TVB's traiting system, which was inspired by the traiting system developed by Enthought (Enthought, 2001). The traiting system of TVB, among other things, provides a mechanism for annotating data, that is, associating additional information with the data which is itself usually 
a single number or an array of numbers. A complete description of TVB's traiting system is beyond the scope of this article. However, in describing TVB's Datatypes we will give an example of its use, which should help to provide a basic understanding of the mechanism.

A number of basic TVB-Datatypes are defined based on Types that are part of the traiting system, with these traited Types, in turn, wrapping Numpy data types. For instance, TVB-FloatArray is a datatype derived from the traiting system's Array type, which in turn wraps Numpy's ndarray. The traiting system's Array type has attributes or annotations, such as: dtype, the numerical type of the data contained in the array; label, a short (typically one or two word) description of what the Array refers to, this information is used by the supporting framework to create a proper label for the GUI; doc, a longer description of what the Array refers to, allowing the direct integration of useful documentation into array objects; and default, the default value for an instance of an Array type. In the case of a FloatArray, the dtype attribute is fixed as being numpy.float64.

More complex, higher-level, TVB-Datatypes are then built up with attributes that are themselves basic TVB-Datatypes. For example, TVB-Connectivity is datatype which includes multiple FloatArrays, as well as a number of other traited types, such as Integer and Boolean, in its definition. An example of a FloatArray being used to define an attribute of a Connectivity can be seen in Code 1. The high-level Datatypes currently defined in TVB are summarized in Table 2.

An example indicating the usage and features of TVBDatatypes is provided below. When a user uploads a connectivity dataset through the UI, an instance of a Connectivity datatype is generated. This Connectivity datatype is one of the required input arguments when creating an instance of SIMULATOR. As a result of the execution of a simulation, other TVB-Datatypes are generated, for instance one or more TimeSeries datatypes. Specifically, if the simulation is run using the MEG and EEG recording modalities then TimeSeriesMEG, TimeSeriesEEG, which are subclasses of TimeSeries, are returned. Both the Connectivity

Code 1 | An instance of TVB's FloatArray Datatype being used to define the conduction speed between brain regions as an attribute of a Connectivity Datatype.

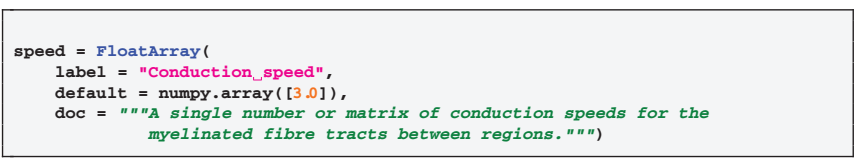

Table 2 | TVB Datatypes.

\begin{tabular}{|c|c|c|}
\hline Base class datatype & Description & Derived classes \\
\hline Connectivity & Maps connectivity matrix data & Connectivity \\
\hline Surfaces & Covers surface representations & $\begin{array}{l}\text { CorticalSurface, SkinAir, BrainSkull, SkullSkin, } \\
\text { EEGCap, FaceSurface, Cortex, RegionMapping, } \\
\text { LocalConnectivity }\end{array}$ \\
\hline Volumes & Wraps volumetric data & ParcellationMask, StructuralMRI \\
\hline Sensors & $\begin{array}{l}\text { Wraps sensors data used in different acquisition techniques to } \\
\text { generate physiological recordings }\end{array}$ & SensorsEEG, SensorsMEG, SensorsInternal \\
\hline \multirow[t]{3}{*}{ ProjectionMatrix } & $\begin{array}{l}\text { Wraps matrices defining a linear operator to map the spatial sources } \\
\text { into the leadfield domain }\end{array}$ & $\begin{array}{l}\text { ProjectionRegionEEG, ProjectionSurfaceEEG, } \\
\text { ProjectionRegionMEG }\end{array}$ \\
\hline & $\begin{array}{l}\text { It relates two datatypes: a source of type Connectivity or Surface } \\
\text { and a set of Sensors }\end{array}$ & ProjectionSurfaceMEG \\
\hline & The matrix is computed using OpenMEEG. (Gramfort et al., 2010) & \\
\hline Equations & $\begin{array}{l}\text { Commonly used functions for defining local connectivity kernels and } \\
\text { stimulation patterns }\end{array}$ & \\
\hline SpatialPattern & $\begin{array}{l}\text { Contains patterns mainly used as stimuli. It makes use of Equation } \\
\text { datatypes }\end{array}$ & $\begin{array}{l}\text { SpatioTemporalPattern, StimuliRegion, } \\
\text { StimuliSurface, SpatialPatternVolume }\end{array}$ \\
\hline TimeSeries & $\begin{array}{l}\text { One of the most important TVB-Datatypes. Derived classes wrap } \\
\text { measurements recorded under different acquisition modalities }\end{array}$ & $\begin{array}{l}\text { TimeSeriesRegion, TimeSeriesSurface, } \\
\text { TimeSeriesVolume, TimeSeriesEEG, TimeSeriesMEG }\end{array}$ \\
\hline Graph & $\begin{array}{l}\text { Wraps results from a covariance analysis or results from BCT } \\
\text { analyzers }\end{array}$ & Covariance, ConnectivityMeasure \\
\hline
\end{tabular}

MappedValues Wraps a single value computed from a TimeSeries object

ModeDecomposition Wraps results from matrix factorization analysis (i.e., PCA and ICA)

PrincipalComponents, IndependentComponents

Spectral Wraps results from frequency analysis

FourierSpectrum, WaveletCoefficients,

ComplexCoherenceSpectrum

Specifications about the requirements to build a TVB-Datatype can be found in the documentation of the distribution packages. 
and TimeSeries datatypes are accepted by a range of appropriate analysis and visualization methods.

Further, TVB-Datatypes have attributes and metadata which remains accessible after exporting in TVB format. The metadata includes a technical description of the data (storage size for instance) as well as scientifically relevant properties and useful documentation to properly interpret the dataset. In the shell interface, the attributes of TVB-Datatype can be accessed by their key-names in the same way as Python dictionaries.

\subsection{TVB SIMULATOR}

The simulation core of TVB brings together a mesoscopic model of neural dynamics with structural data. The latter defines both the spatial support (see Figure 4), upon which the brain network model is built, and the hierarchy of anatomical connectivity, that determines the spatial scale represented by the structural linkages between nodes (Freeman, 1975). Simulations then recreate the emergent brain dynamics by numerically integrating this coupled system of differential equations. All these entities have their equivalent representation as classes either in the scientific MODULES or datatypes, and are bound together in an instance of the Simulator class. In the following paragraphs we describe all the individual components required to build a minimal representation of a brain network model and run a simulation, as well as the outline of the operations required to initialize a Simulator object and the operations of the update scheme.

\subsubsection{Coupling}

The brain activity (state variables) that has been propagated over the long-range Connectivity pass through these functions before entering the equations of a Model describing the local dynamics. A Coupling function's primary purpose is to rescale the incoming activity to a level appropriate to the population model. The base Coupling class as well as a number of different coupling functions are implemented in the COUPLING module, for instance Linear and Sigmoidal.

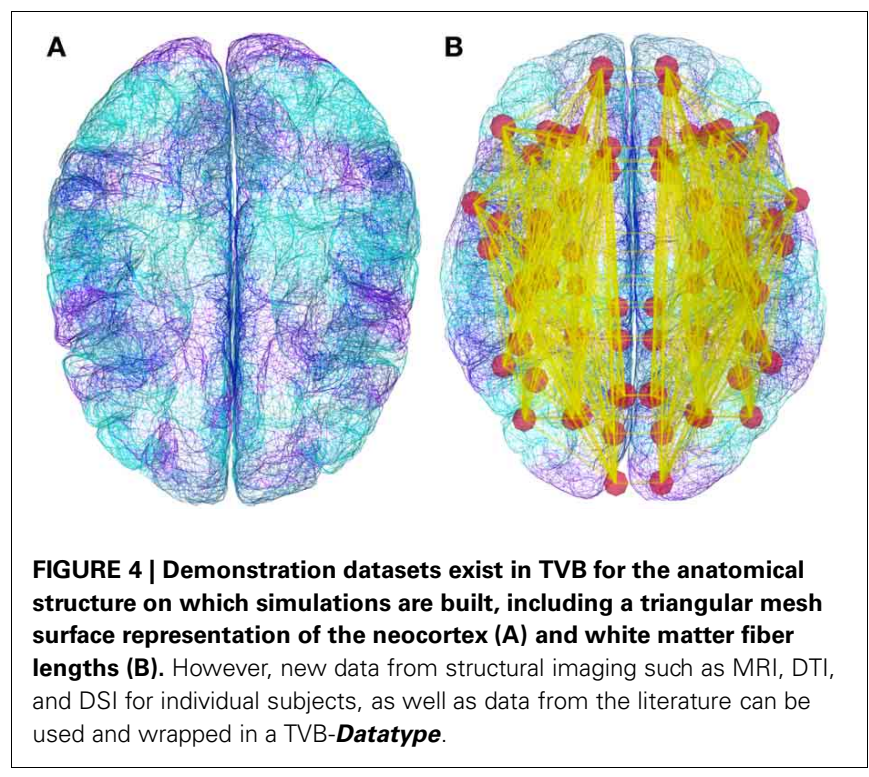

\subsubsection{Population models}

A set of default mesoscopic neural models are defined in TVB's MODELS. All these models of local dynamics are classes derived from a base Model class.

We briefly discuss the implemented population models in order of increasing complexity. They include a generic two dimensional oscillator, a collection of classical population models and two recently developed multi-modal neural mass models. Below, $N$ refers to the number of state variables or equations governing the evolution of the model's temporal dynamics; $M$ is the number of modes and by default $M=1$ except for the multi-modal models.

The Generic2doscillator model $(N=2)$ is a generic phase-plane oscillator model capable of generating a wide range of phenomena observed in neuronal population dynamics, such as multistability, the coexistence of oscillatory and non-oscillatory dynamics, as well as displaying dynamics at multiple time scales.

The WilsonCowan model (Wilson and Cowan, 1972) $(N=2)$ describes the firing rate of a neural population consisting of two subpopulations (one excitatory and the other inhibitory). It was originally derived using phenomenological arguments. This neural mass model provides an intermediate between a microscopic and macroscopic level of description of neural assemblies and populations of neurons since it can be derived from pulse-coupled neurons (Haken, 2001) and its continuum limit resembles neural field equations (Jirsa and Haken, 1996).

The WongWang model (Wong and Wang, 2006) represents a reduced system of $N=2$ coupled non-linear equations, originally derived for decision making in two-choice tasks. The BrunelWang model (Brunel and Wang, 2001, 2003) is a mean field model derived from integrate-and-fire spiking neurons and makes the approximation of randomly distributed interspike intervals. It is notable that this population model shows only attractor states of firing rates. It has been extensively used to study working memory. Its complexity resides in the number of parameters that it uses to characterize each population $(N=2)$. These parameters correspond to physical quantities that can be measured in neurophysiology experiments. The current implementation of this model is based on the approach used in (Deco and Jirsa, 2012).

The JansenRit model (Jansen and Rit, 1995) is a derivative of the Wilson-Cowan model and features three coupled subpopulations of cortical neurons: an excitatory population of pyramidal cells interacting with two populations of interneurons, one inhibitory and the excitatory. This model can produce alpha activity consistent with that measured in EEG, and is capable of simulating evoked potentials (Jansen et al., 1993). It displays a surprisingly rich and complex oscillatory dynamics under periodic stimulation (Spiegler et al., 2010). Each population is described by a second order differential equation. As a consequence the system is described by a set of $N=6$ first order differential equations.

The StefanescuJirsa2D and StefanescuJirsa3D models (Stefanescu and Jirsa, 2008; Jirsa and Stefanescu, 2010; Stefanescu and Jirsa, 2011) are neural mass models derived from a 
globally coupled population of neurons of a particular kind. The first one has been derived from coupled FitzHugh-Nagumo neurons (FitzHugh, 1961; Nagumo, 1962), which, with $N=2$, are capable of displaying excitable dynamics, as well as oscillations. The second is derived from coupled Hindmarsh-Rose neurons (Hindmarsh and Rose, 1984), which are also capable of producing excitable and oscillatory dynamics, but with $N=3$ have the additional capability of displaying transient oscillations and bursts. The two Stefanescu-Jirsa models show the most complex repertoire of dynamics (including bursting and multi-frequency oscillations). They have been derived using mean field techniques for parameter dispersion (Assisi et al., 2005) and have an additional dimension, the mode $M$, which partitions the dynamics into various subtypes of population behavior. These models are therefore composed of $12(N=4, M=3)$ and $18(N=6$, $M=3$ ) state variables, respectively.

\subsubsection{Integrators}

The base class for integration schemes is called Integrator, an INTEGRATORS module contains this base class along with a set of specific integration scheme classes for solving both deterministic and stochastic differential equations. The specific schemes implemented for brain network simulations include the Euler and Heun methods. The 4th-order Runge-Kutta (rk4) method is only available for solving ordinary differential equations (ODEs), i.e., deterministic integration, given that there are various variants for the stochastic version of the method, differing rates of convergence being one of the points that several attempts of creating a stochastic adaptation fail at [see Burrage et al. (2004) for an overview]. Therefore, this method is available for drawing example trajectories in the interactive phase-plane plot tool.

\subsubsection{Noise}

Noise plays a crucial role for brain dynamics, and hence for brain function (McIntosh et al., 2010). The NOISE module consists of two base classes: RandomStream that wraps Numpy's RandomState class and Noise. The former provides the ability to create multiple random streams which can be independently seeded or set to an explicit initial state. The latter is the base class from which specific noises, such as white and colored (Fox et al., 1988), are derived. In TVB's implementation Noise enters as an additional term within the stochastic integration schemes, and can be either an Additive or Multiplicative process (Klöden and Platen, 1995). As well as providing a means to generate reproducible stochastic processes for the integration schemes, the related classes in NOISE are used to set the initial conditions of the system when no explicit initial conditions are specified.

\subsubsection{Monitors}

The data from a simulation is processed and recorded while the simulation is running, that is, while the differential equations governing the system are being integrated. The base class for these processing and recording methods is the Monitor class in the MONITORS module. We consider two main types of online-processing: (1) raw or low-level; and (2) biophysical or high-level. The output of a Monitor is a 4-dimensional array (which can be wrapped in the corresponding TimeSeries datatype), i.e., a $3 \mathrm{D}$ state vector as a function of time. For the first kind of Monitors these dimensions correspond to [time, state variables, space, modes] where "space" can be either brain regions or vertices of a cortical surface plus non-cortical brain regions. The number of state variables as well as the number of modes strictly depend on the Model. For the second kind of Monitors, the dimensions are [time, 1, sensors, 1]. The simplest form of low-level Moni tor returns all the simulated data, i.e., time points are returned at the sampling rate corresponding to the integration scheme's step size and all state variables are returned for all nodes. All other low-level Monitors perform some degree of down-sampling, such as returning only a reduced set state variables (by default the variables of interest of a Mode1), or down-sampling in "space" or time. Some variations include temporally sub-sampled, spatially averaged and temporally sub-sampled, or temporally averaged. The biophysical Monitors instantiate a physically realistic measurement process on the simulation, such as EEG, MEG, SEEG or BOLD. For the first two, a ProjectionMatrix is also required. This matrix maps source activity ("space") to sensor activity ("sensors"). OpenMEEG (Gramfort et al., 2010) was used to generate the demonstration projection matrix, also known as lead-field or gain matrix, that corresponds to the EEG/MEEG forward solution. The forward solution modeling the signals from depth electrodes is based on the point dipole model in homogeneous space (Sarvas, 1987). The BOLD monitor is based on Buxton and Frank (1997) and Friston et al. (2000). Figure 5 summarizes the fundamental blocks required to configure a full model, launch a simulation and retrieve the simulated data.

In most neural mass models there is a state variable representing some type of neural activity (firing rate, average membrane potential, etc.), which serves as a basis for the biophysical monitors. The state variables used as source of neural activity depend both on the Model and the biophysical space that it will be projected onto (MEG, EEG, BOLD). Given a neural mass model with a set of state variables, G-Users can choose which subset of state variables will be fed into a Monitor (independently for each monitor). However, how a given Monitor operates on this subset of state variables is an intrinsic property of the monitor. Users with programming experience can, of course, define new monitors according to their needs. Currently, there is not a mechanism providing automatic support for general operations over state variables before they are passed to a monitor. As such, when the neural activity entering into the monitors is anything other than a summation or average over state variables then it is advised to redefine the Model in a way that one of the state variables actually describes the neural activity of interest.

\subsubsection{Outline of the simulation algorithm}

The Simulator class has several methods to set up the spatiotemporal dimensions of the input and output arrays, based on configurable attributes of the individual components such as integration time step (e.g., INTEGRATORS.HeunDeterministic.dt), structural spatial support (e.g., connectivity.Connectivity or surfaces. CorticalSurface) and transmission speed (e.g., connectivity.Connectivity.speed) as well as a cascade of specific 


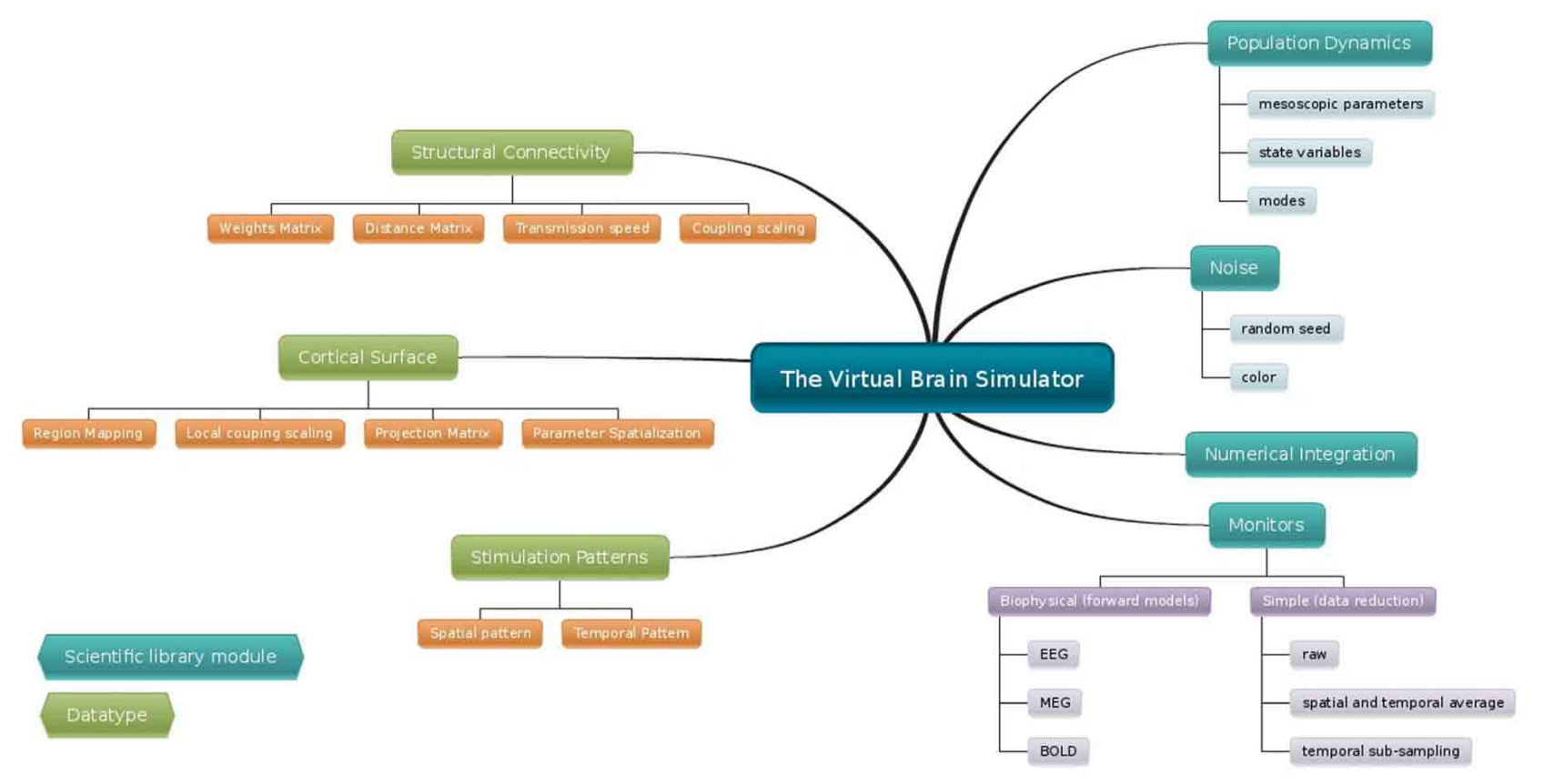

FIGURE 5 | Diagram of the configurable elements for building a brain network model and launching a simulation. TVB can incorporate cortical connectivity information from an individual's tractographic and cortical geometry data. The Connectivity object contains matrices defining the connection strengths and time delays via finite signal transmission speed between all regions, while the folded Cortical Surface mesh provides the spatial support for finer resolution models. In the latter case a Local Coupling defines the interaction between neighboring nodes. In its simplest form local connectivity is spatially invariant, however, support exists for spatial inhomogeneity. Signal propagation via local connectivity is instantaneous (no time delays), which is a reasonable approximation considering the short distances involved. Together, the cortical surface with its local connectivity, the long-range connectivity matrix, and the neural mass models defining the local dynamics define a full brain network model. Additionally, stimulation can be applied to a simulation. The stimulation patterns are built in terms of spatial and temporal equations chosen independently. For region-based network models, it is only possible to build time dependent stimuli since there is not a spatial extent for a region node. However, node-specific weightings can be set to modulate the intensity of the stimulus applied to each node. For surface-based models, equations with finite spatial support are evaluated as a function of distance from one or more focal points (vertices of the surface), where the equation defines the spatial profile of the stimuli. The neural source activity from both region or surface-based approaches can be projected into EEG, MEG and BOLD (Buxton and Frank, 1997; Friston et al., 2000) space using a forward model (Breakspear and Jirsa, 2007). configuration methods to interface them. The Simulator class coordinates the collection of objects from all the modules in the scientific library needed to build the network model and yield the simulated data. To perform a simulation a Simulator object needs to be: (1) configured, initializing all the individual components and calculating attributes based on the combination of objects passed to the Simulator instance; and (2) called in a loop to obtain simulated data, i.e., to run the simulation (see Code 2). The next paragraphs list the main operations of the simulation algorithm.

\section{Initializing a Simulator}

1. Check if the transmission speed was provided.

2. Configure the Connectivity matrix (connectome). The delays matrix is computed using the distance matrix and the transmission speed. Get the number of regions.

3. Check if a Surface is provided.

4. Check if a stimulus pattern is provided.

5. Configure individual components: Model, Integrator, Monitors. From here we obtain integration time step size, number of statevariables, number of modes.

6. Set the number of nodes (region-based or surface-based simulation). If a Surface was given the number of nodes will correspond to the number of vertices plus the number of non-cortical regions, otherwise it will be equal to the number of regions in the Connectivity matrix.

7. Spatialise model parameters if required. Internally, TVB uses arrays for model parameters, if the size of the array for a particular parameter is 1 , then the same numerical value is applied to all nodes. If the size of the parameter array is $N$, where $N$ is the number of nodes, the parameter value for each node is taken from the corresponding element of the array of parameter values.

8. If applicable, configure spatial component of stimulation Patterns (requires number of nodes).

9. Compute delays matrix in integration time steps.

10. Compute the horizon of the delayed state, that is the maximum delay in integration time steps.

11. Set the history shape. The history state contains the activity that propagates from the delayed state to the next. 
12. Determine if the Integrator is deterministic or stochastic. If the latter, then configure the Noise and the integration method accordingly.

13. Set initial conditions. This is the state from which the simulation will begin. If none is provided, then random initial conditions are set based on the ranges of the model's state variables. Random initial conditions are fed to the initial history array providing the minimal state of the network with time-delays before $t=0$. If initial conditions are user-defined but the length along the time dimension is shorter than the required horizon, then the history array will be padded using the same method of described for random initial conditions.

14. Configure the monitors for the simulation. Get variables of interest.

\section{Calling a simulator}

1. Get simulation length.

2. Compute estimates of run-time, memory usage and storage.

3. Check if a particular random state was provided (random seed). This feature is useful for reproducibility of results, for instance, getting the same stream of random numbers for the Noise.

4. Compute the number of integration steps.

5. If the simulation is surface-based, then get attributes required to compute Local Connectivity kernel.

6. Update state loop:

a. Get the corresponding coupled delayed activity. That is, compute the dot product between the weights matrix (connectome) and the delayed state of the coupling variables, transformed by a (long-range) coupling function.

b. Update the state array. This is the numerical integration, i.e., advancing an integration time step, of the differential equations defining the neuron model. Distal delayed activity, local instantaneous activity and stimulation are fed to the integration scheme.

c. Update the history.

d. Push state data onto the Monitors. Yield any processed time-series data point if available.

As a working example, in Code 2, we show a code snippet which uses TVB's scripting interface and some of the classes and modules we have just described to generate one second of brain activity. The for loop in the example code allows scripting users to receive time-series data as available and separately for each of the monitors processing simulated raw data. In this implementation, at each time step or certain number of steps, data can be directly stored to disk, reducing the memory footprint of the simulation. Such a feature is particularly useful when dealing with larger simulations. Likewise, data can be accessed while the simulation is still running, which proves to be advantageous for modeling paradigms where one of the output signals is fed back to the network model as stimulation for instance (see the paragraph about Dynamic modeling in section 3).
Code 2 | Script example to simulate 1 second of brain activity. Output is recorded with two different monitors.

\begin{tabular}{|c|}
\hline 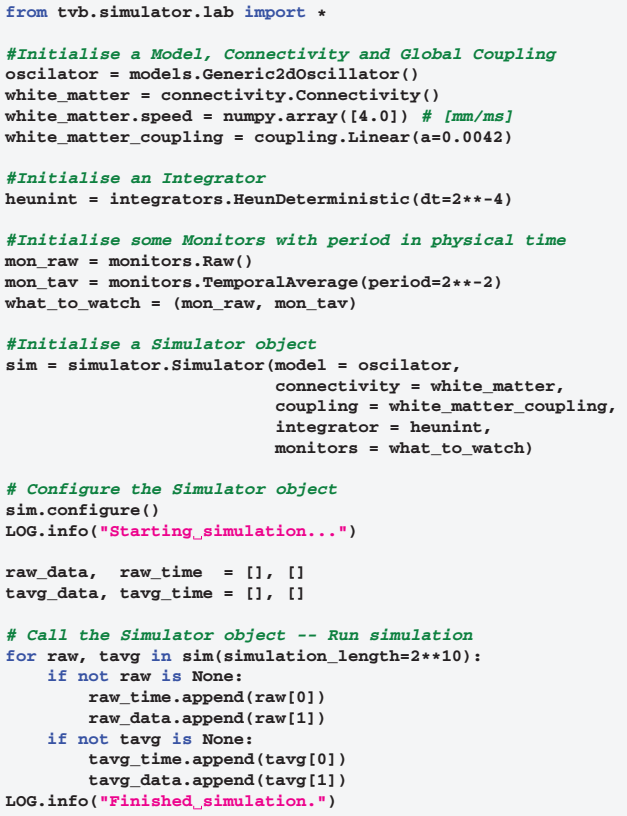 \\
\hline
\end{tabular}

\subsection{ANALYZERS AND VISUALIZERS}

For the analysis and visualisation of simulated neuronal dynamics as well as imported data, such as anatomical structure and experimentally recorded time-series, several algorithms and techniques are currently available in TVB. Here we list some of the algorithms and methods that are provided to perform analysis and visualization of data through the GUI.

Analyzers are mostly standard algorithms for time-series and network analysis. The analyzers comprise techniques wrapping functions from Numpy (Fast Fourier Transform (FFT), autocorrelation, variance metrics), Scipy (cross-correlation), scikitlearn (ICA) (Pedregosa et al., 2011) and matplotlib-mlab (PCA) (Hunter, 2007). In addition, there are specific implementations of the wavelet transform, complex coherence (Nolte et al., 2004; Freyer et al., 2012) and multiscale entropy (MSE) (Costa et al., 2002, 2005; Lake and Moorman, 2011).

Visualizers are tools designed to correctly handle specific datatypes and display their content. Representations currently available in the GUI include: histogram plots (Figure 6A); interactive time-series plots, EEG (Figure 6C); 2D head topographic maps (Figure 6B); 3D displays of surfaces and animations (Figure 6D) and network plots. Additionally, for shell users there is a collection of plotting tools available based on matplotlib and mayavi (Ramachandran and Varoquaux, 2011).

\section{PERFORMANCE, REPRODUCIBILITY, AND FLEXIBILITY 3.1. TESTING FOR SPEED}

In the context of full brain models there is no other platform against which we could compare the performance results for TVB 


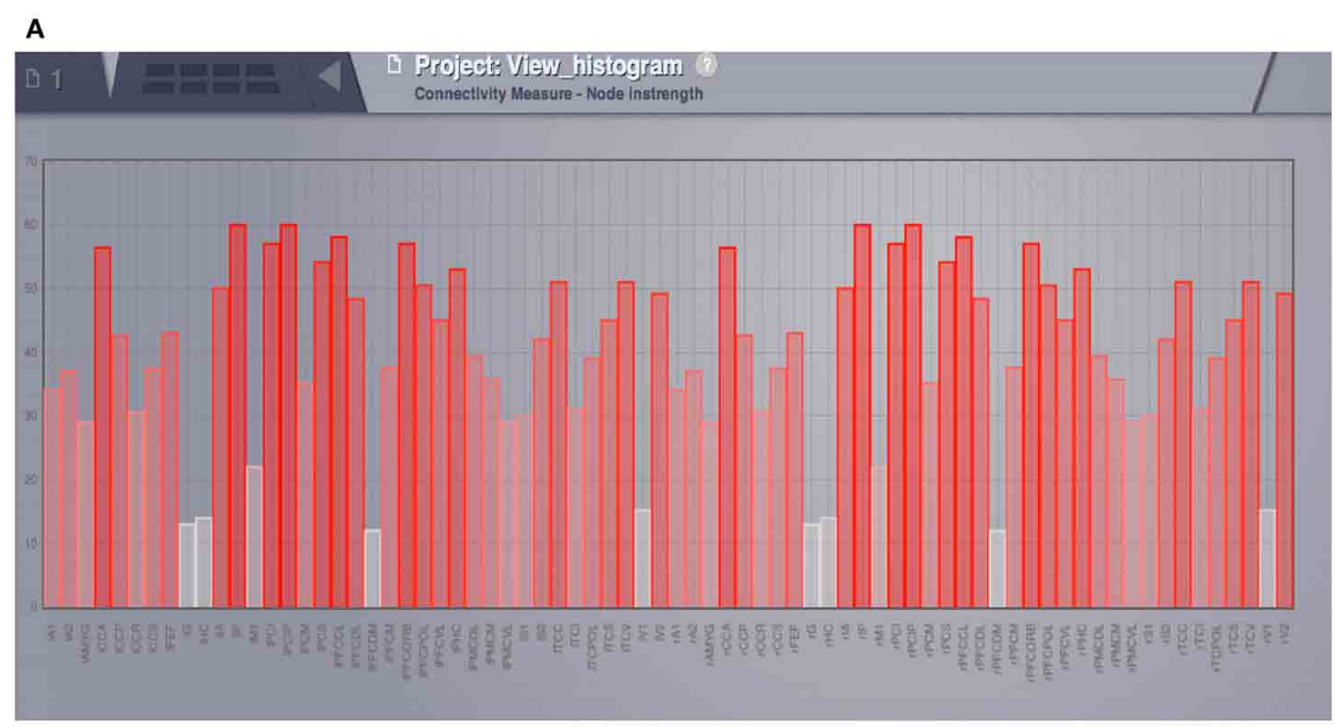

B

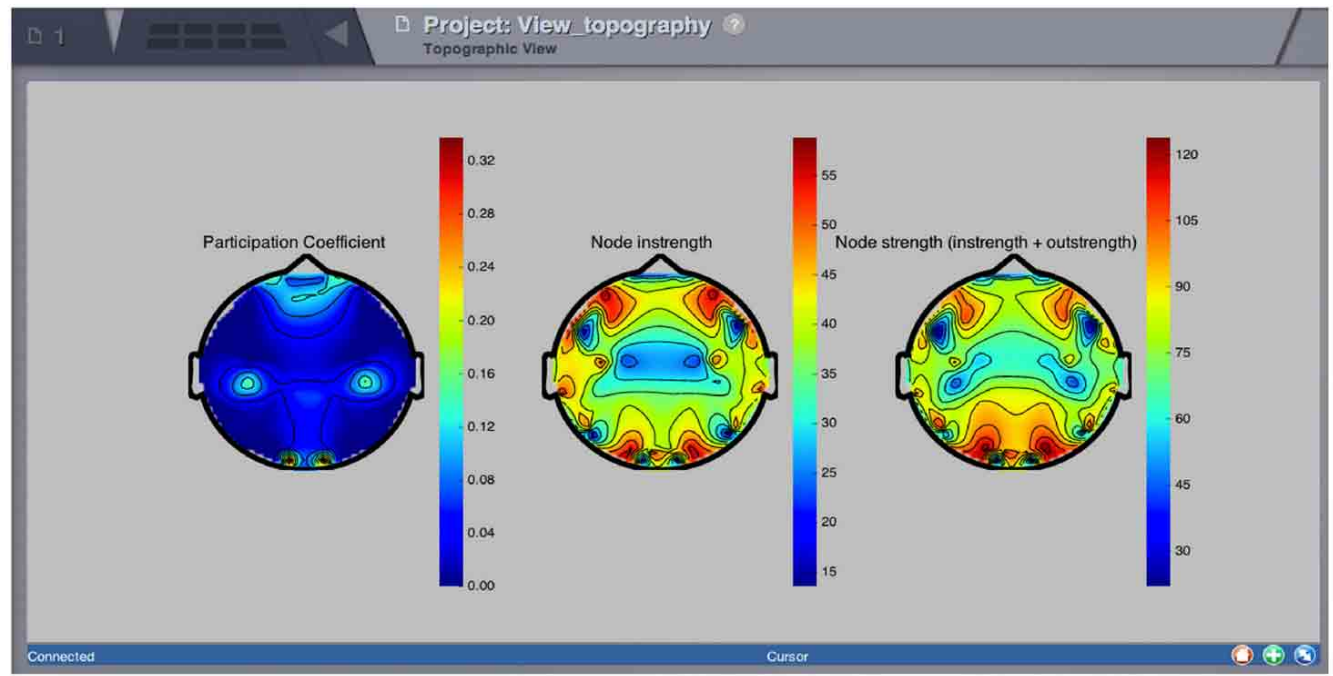

C

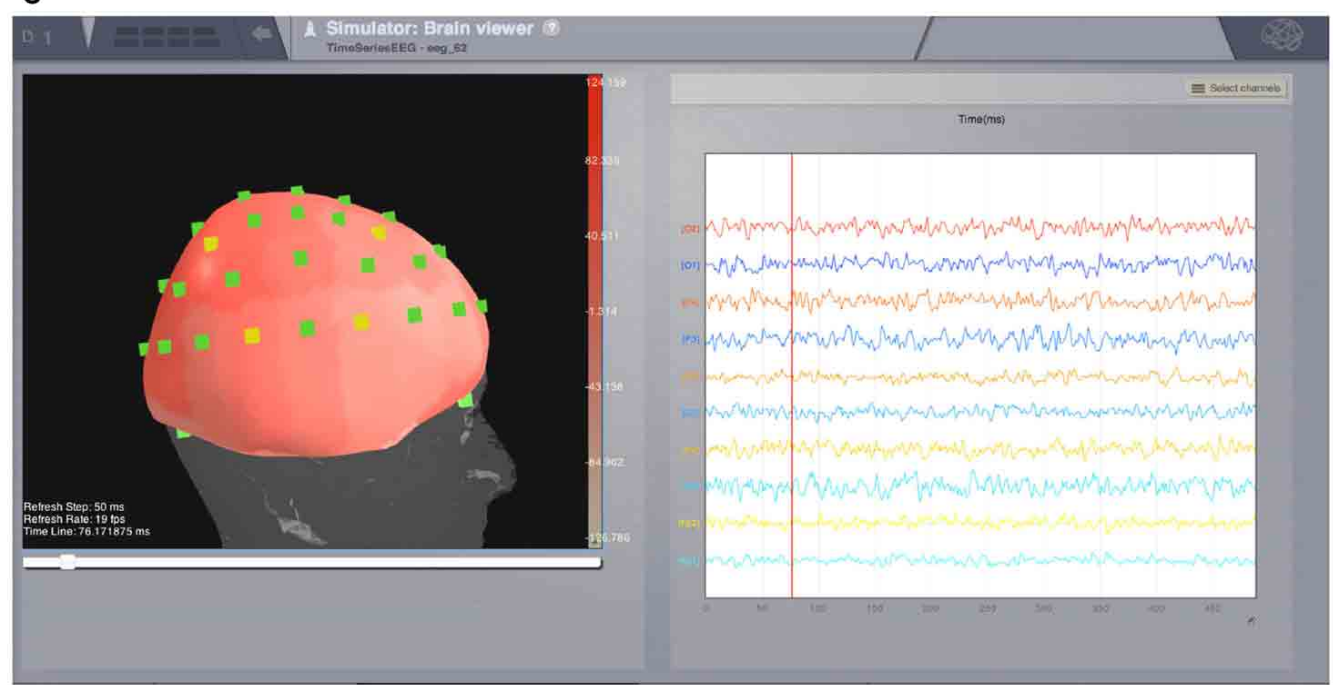

FIGURE 6 | Continued 


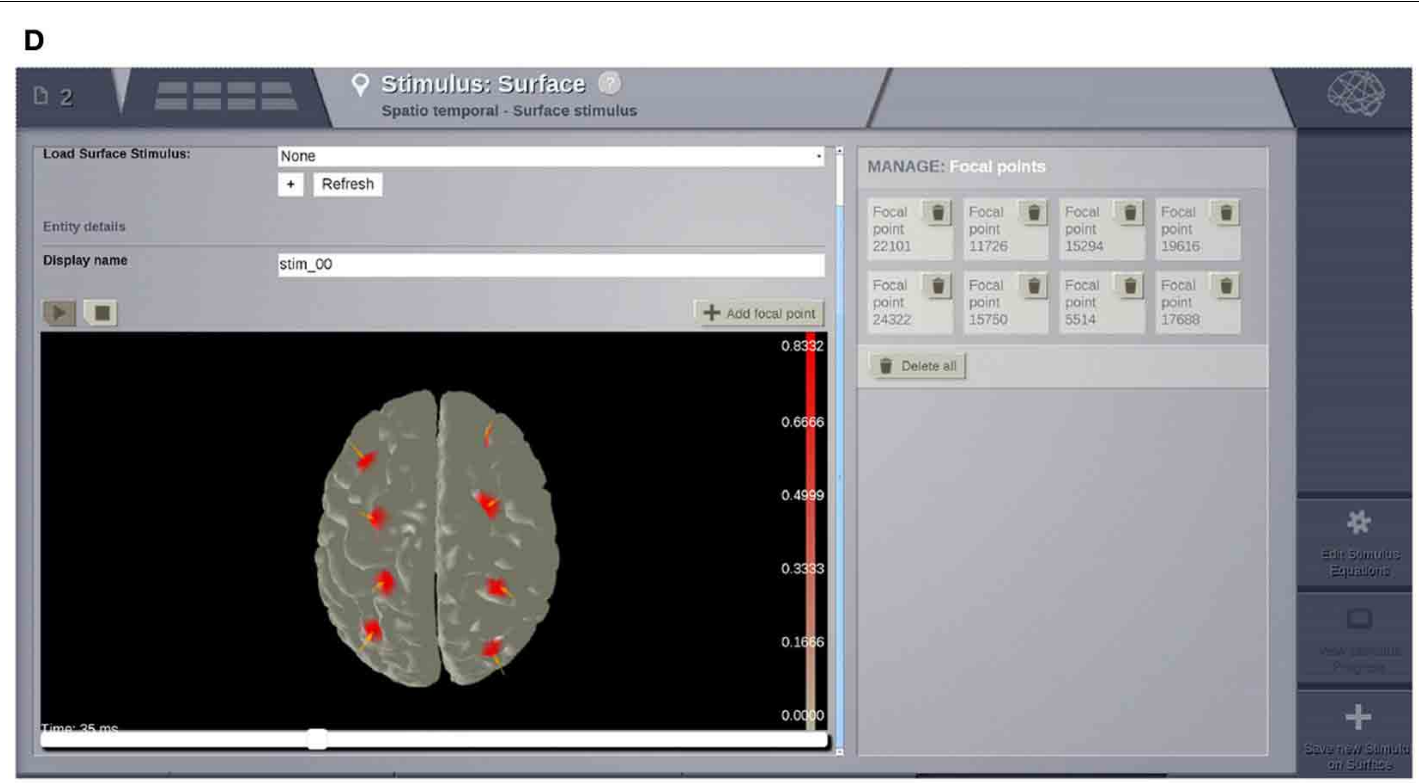

FIGURE 6 | Visualizers. (A) Histogram of a graph metric as a function of nodes in the connectivity matrix. (B) A 2D projection of the head. The color map represents a graph metric computed on the connectivity matrix. (C) EEG visualizer combines a rendered head surface, an overlay with the sensors positions and an interactive time-series display. (D) An animated display of the spatiotemporal pattern applied to the cortical surface. Red spots represent the focal points of the spatial component of the stimulus. and define a good ratio run-time/real-time. As a first approximation a simple network of 74 nodes, whose node dynamics were governed by the equations of the Generic2doscillator model (see Code 3) was implemented in the Brian spiking neural network simulator. The integration step size was $0.125 \mathrm{~ms}\left(d t=2^{-3} \mathrm{~ms}\right)$ and the simulation length was $2048 \mathrm{~ms}$. This network was evaluated without time delays and using a random sparse connectivity matrix. Execution times were about $4.5 \mathrm{~s}$ in Brian and $15 \mathrm{~s}$ in TVB. In contrast, when heterogeneous time delays were included, running times of the simulations implemented in Brian increased considerably (approximately 6.5x) whereas in TVB they hardly changed (approximately $1.2 \mathrm{x}$ ). Simulations were run on a CPU Intel ${ }^{\circledR}$ Xeon ${ }^{\circledR}$ W3520@ $2.67 \mathrm{GHz}$. These results, although informative, expose the fact that the architectures of TVB and the Brian simulator are different and therefore they have been optimized accordingly to serve distinct purposes from a modeling point of view.

To assess the performance of TVB in terms of simulation timings, we also ran simulations for all possible combinations of two parameters: simulation length and integration time step (Figure 7A). We made the following estimates: it takes on average $16 \mathrm{~s}$ to compute $1 \mathrm{~s}$ of brain network dynamics [at the region level, with an integration time step of $0.0625 \mathrm{~ms}\left(d t=2^{-4} \mathrm{~ms}\right)$ and including time delays of the order of $20 \mathrm{~ms}$ which amounts to store about 320 past states per time step] on CPUs Intel ${ }^{\circledR}$ Xeon ${ }^{\circledR X} 5672$ @ $3.20 \mathrm{GHz}, \mathrm{CPU}$ cache of $12 \mathrm{MB}$ and Linux kernel 3.1.0-1-amd64 as operating system. In Figure 7B we quantify how running times increase as a function of the integration time step in $64 \mathrm{~s}$ long (region-based) simulations for two different sizes of the connectivity matrix.
Code 3 | State equations of the generic plane oscillator as scripted to run the simulation in the Brian simulator. The description of the parameters are explained in the API documentation and will be discussed in the context of dynamical systems elsewhere.

\# model equations
eqs $=\ldots$
$d V / d t=d * t a u *(a l p h a * W-f * V * * 3+e * V * * 2+I)$
$d W / d t=d *(a+b * V+c * V * * 2-b e t a * W) / \operatorname{tau}$
$\prime \prime$

In general, human cortical connectomes are derived from anatomical parcellations with a variable number of nodes, from less than 100 to over a few thousands nodes (Zalesky et al., 2010). Preliminary results of simulations (data not shown) using connectivity matrices of different sizes $(16,32,64,128,256,512$, 1024, 2048, and 4096 nodes) and a supplementary parameter (transmission speed that has an effect on the size of the history array keeping the delayed states of the network) indicate that there is a quadratic growth of the running times for networks with more than 512 nodes. Since performance depends on a large number of parameters which have an effect on both memory (CPU cache and RAM) and CPU usage, and therefore resulting running times arise from the interaction between them, we see the need to develop more tests to stress in particular memory capacity and bandwidth in order to fully understand the aforementioned behavior.

In Future Work we talk about the approaches to benchmark and improve the execution times of simulations. For the present work we have restricted ourselves to present performance results looking at the parameters that have the strongest effect on simulations timings. 


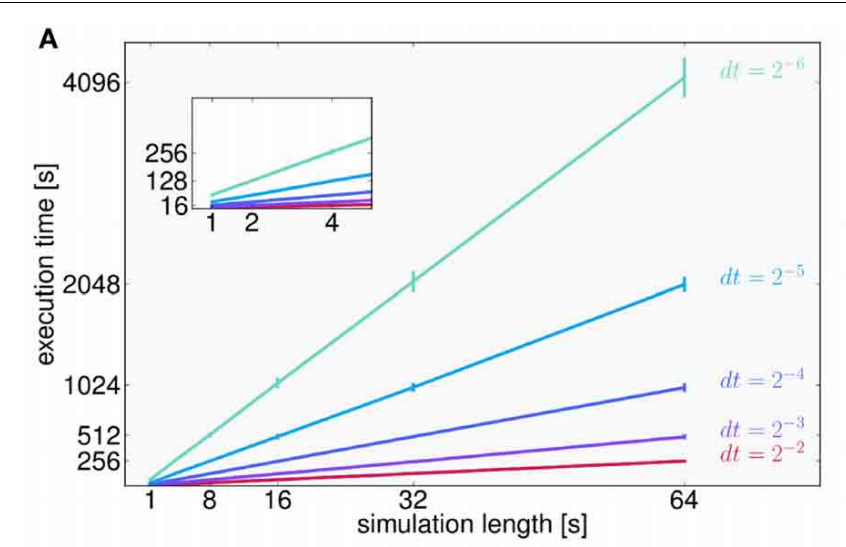

FIGURE 7 | (A) As expected for fixed time-step schemes, execution times scale linearly with the number of integration steps. We used seven values of simulation lengths $(1,2,4,8,16,32$, and $64 \mathrm{~s})$ and five values of integration time step $\left(d t=2^{-2}=0.25, d t=2^{-3}=0.125\right.$, $d t=0.0625=2^{-4}, d t=0.03125=2^{-5}$, and $d t=0.015625=2^{-6} \mathrm{~ms}$ ). For each possible combination 100 simulations were performed. The network model consisted of 74 nodes (with two state variables and one mode per node). Numerical integration was based on Heun's stochastic method. We plot the average execution time with the error bars representing the standard deviation over simulations. The inset shows a narrower range for simulation lengths between 1 and $4 \mathrm{~s}$. Axes units

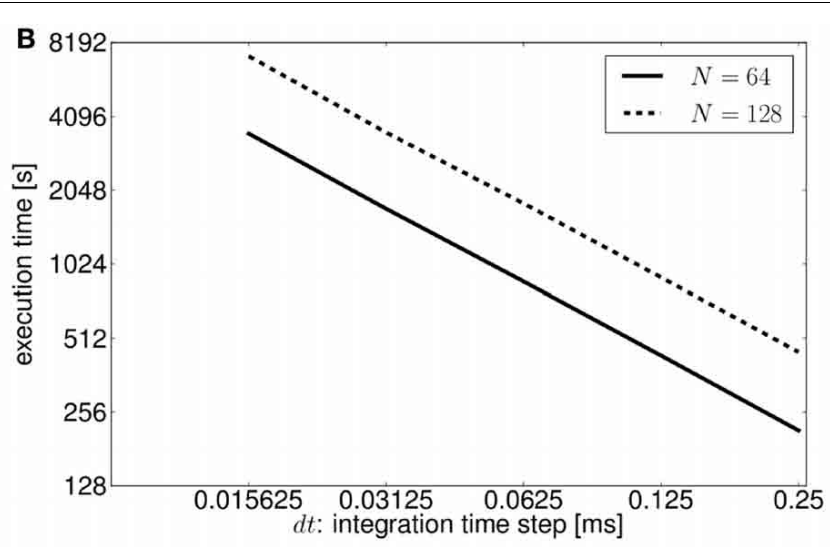

and color code are the same as those displayed in the main plot. (B) Here, execution times are shown as a function of the integration time step size, $d t$, for two different number of nodes (solid and dashed lines correspond to connectivity matrices of 64 and 128 nodes, respectively) for a specific conduction speed $(4 \mathrm{~mm} / \mathrm{ms})$ and simulation length $(64 \mathrm{~s})$. Both axes are in logarithmic scale with base 2. In this case, halving $d t$ or doubling the number of nodes in the connectivity matrix, $N$, doubles the running time. However, as mentioned in the text, for larger networks execution times seem to grow quadratically as a function of the number of nodes in the network. Further tests need to be developed to understand this behavior.

\subsection{REPRODUCIBILITY OF RESULTS FROM THE LITERATURE}

Ghosh et al. (2008) and Deco et al. (2009) demonstrated the important role of three large-scale parameters in the emergence of different cluster synchronization regimes: the global coupling strength factor, time-delays (introduced via the long-range connectivity fiber tract lengths and a unique transmission speed) and noise variance. They built parameter space maps using the Kuramoto synchronization index. Here, using TVB's scripting interface, we show it is easily possible to build a similar scheme and perform a parameter space exploration in the coupling strength ( $g c s)$ and transmission speed (s) space. The Connectivity upon which the large-scale network is built was the demonstration dataset. It is bi-hemispheric and consists of 74 nodes, i.e., 37 regions per hemisphere. It includes all the cortical regions but without any sub-cortical structure such as the thalamic nuclei. Its weights are quantified by integer values in the range $0-3$. The evolution of the local dynamics were represented by the model Generic2dOscillator, configured in such a way that a single isolated node exhibited $40 \mathrm{~Hz}$ oscillations (Figure 8). The variance of the output time-series was chosen as a simple, yet informative measure to represent the collective dynamics (Figure 9A) as a function of the parameters under study. Results are shown in Figure 9B. Parameter sweeps can also be launched from TVB web-interface (see Figure 10 for an illustration).

Currently TVB provides two scalar metrics based on the variance of the output time-series to perform data reduction when exploring a certain parameter space. These are Variance of the nodes Variances and Global Variance. The former zero-centers the output time-series and computes the variance over time of the concatenated time-series of each state variable and mode for each node and subsequently the variance of the nodes variances is computed. This metric describes the variability of the temporal variance of each node. In the latter all the time-series are zero-centered and the variance is computed over all data points contained in the output array.

With this example we intended to expose the possibility to reproduce workflows, i.e., modeling schemes, found in the literature. TVB is a modeling platform providing a means of crossvalidating scientific work by encouraging reproducibility of the results.

\subsection{HIGHER-LEVEL SIMULATION SCENARIOS USING STIMULATION PROTOCOLS}

As one possible use case, we have set up an example based on the scheme used in McIntosh et al. (2010). The goal is to demonstrate how to build stimulation patterns in TVB, use them in a simulation, obtain EEG recordings of both the activity similar to the resting state (RS) and to evoked responses (ER), and finally make a differential analysis of the complexity of the resulting time-series by computing MSE.

In vision neuroscience, the two-stream hypothesis (Schneider, 1969) suggests the existence of two streams of information processing, the ventral and the dorsal stream. In one of these pathways, the ventral stream, the activity from subcortical regions project to $\mathrm{V} 1$ and the activity propagates to the temporal cortices through V2 and V4 (Goodale and Milner, 1992). We systematically stimulated the area corresponding to the primary visual cortex (V1) to demonstrate the functioning of TVB stimulation Patterns and observed how the activity elicited by a periodic rectangular pulse propagates to neighboring regions, especially V2.

Benefiting from TVB's flexibility we show in Figure 11 that it is possible to systematically stimulate a specific brain region 


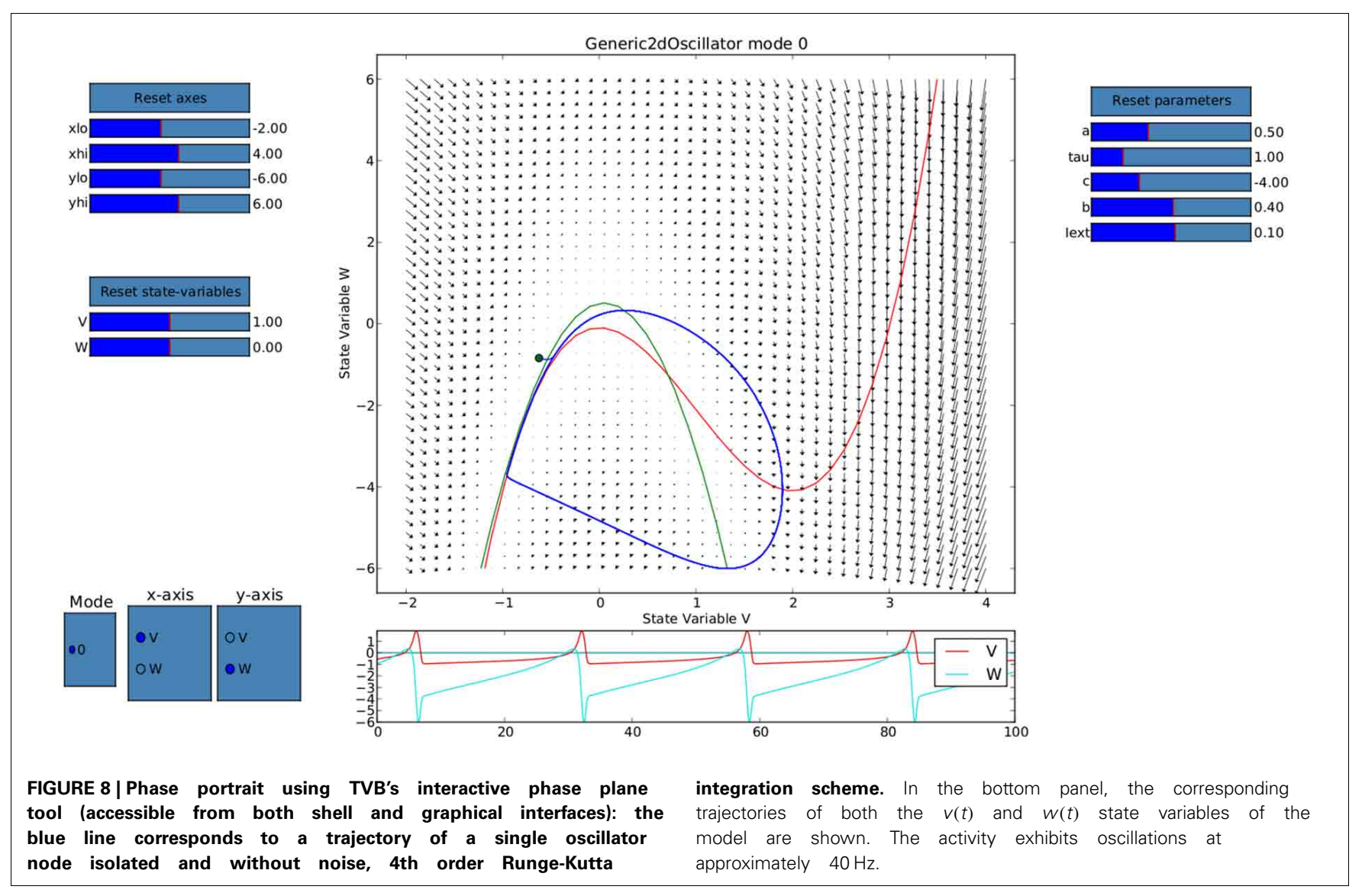

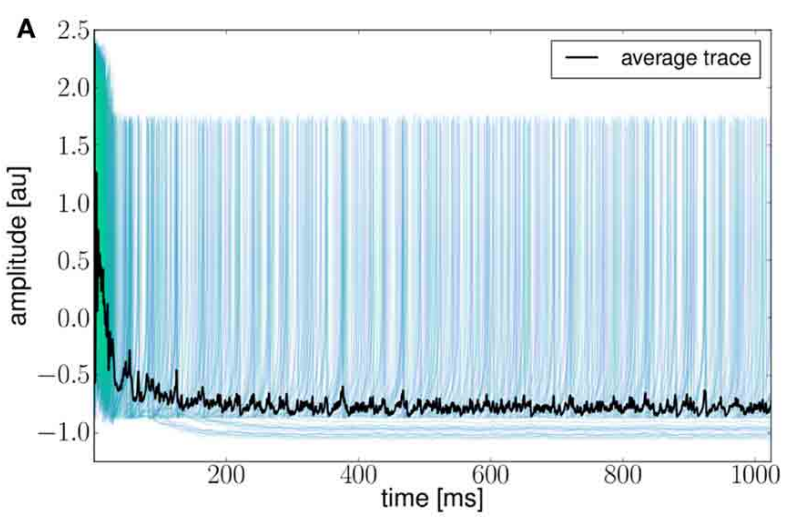

FIGURE 9 | (A) The activity of individual regions are illustrated in colored lines. The black line represents the average activity over the network nodes. Here brain regions are weakly coupled changing both the collective and local dynamics of the network. (B) Using TVB scientific library as a python module we can conveniently run thousands of simulations in parallel on a cluster. Note that TVB parallelizes different tasks e.g., simulations and analyses, taking advantage of multi-core systems, however, it does not parallelize the processes themselves. Simultaneous simulations allow for a systematic

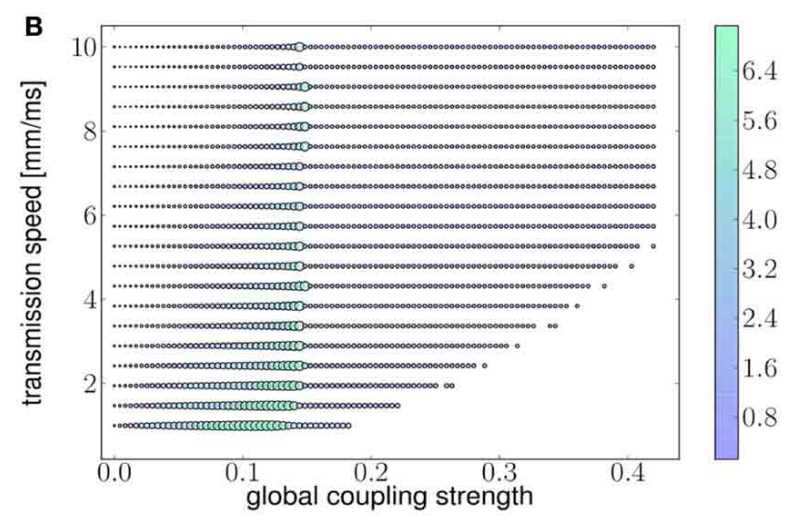

parameter space exploration to rapidly gain insights of the whole brain dynamics repertoire. In this plot, the magnitude and color scale correspond to one the variance computed over all the elements of the $\mathrm{N}$-dimensional output array (Global Variance). Simulations were performed on a cluster based on the Python demo scripts available in the release packages. On of the major strengths of The Virtual Brain is that G-Users are enabled to launch parameter sweeps through the UI without the need to know how to submit parallel jobs (see Figure 10). (e.g., V1) and to highlight the anatomical connection to its target region (e.g., V2) by observing the arrival of the delayed activity; analyze the responses of the model; handle multi-modal simulated data; and extract metrics from computationally expensive algorithms to characterize both the "resting" and "evoked" states.

Currently, TVB permits the stimulation and read-out of activity from any brain area defined in the anatomical parcellation 


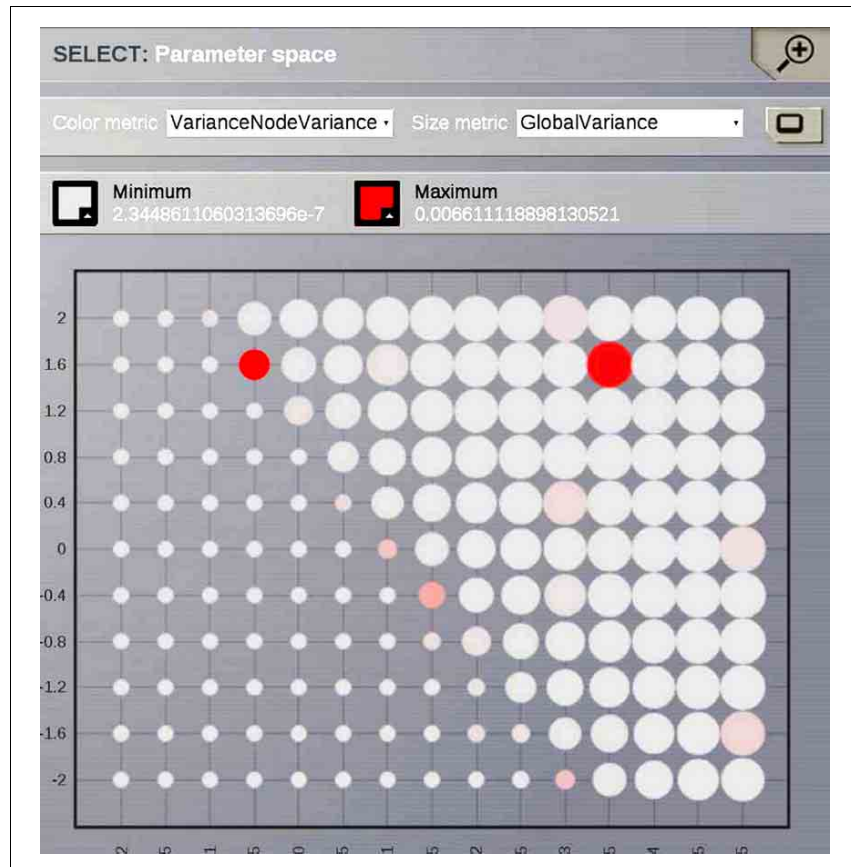

FIGURE 10 | One of TVB's major strengths is the capability to launch parallel simulations through the UI. We show a screenshot of the resulting display when sweeping across two different parameters of the Generic2dOscillator model. Here each data point represents two metrics: size is mapping the Global Variance and color corresponds to the Variance of the nodes Variance. These results provide a topography of the stability space allowing users to distinguish, and thus select, combinations of critical parameters values.

used to derive the connectome. This modeling example was built imposing a strong restriction on the number of regions to stimulate, since global dynamics can quickly become complex. Additionally, to demonstrate the many scenarios that can be set up in TVB, we simulated the same brain network model under the influence of a stimulus, first without noise (Figure 11A: using Heun deterministic method) and then with white noise (Figure 11B: using Heun stochastic method). The first approach makes it easier to see the perturbations induced by the stimulus and the propagation of activity from one region to the other. The second approach is a more realistic representation of the neural activity.

Results of the proposed modeling protocol are presented in Figure 12 where the EEG traces from channel $\mathrm{Oz}$ for the resting and evoked states are shown together with the MSE estimates.

Scripts to reproduce results from Figures 11, 12 are available in the distribution packages of TVB.

With the availability of surface-based simulations the challenge of replicating topographic maps of different sensory systems, such as those found in the primary visual cortex (Hinds et al., 2009), could be addressed.

\subsection{DYNAMIC MODELING}

From both the shell and web interface it is possible to exploit another feature of TVB: namely, simulation continuation, i.e., a simulation can be stopped allowing users to modify model parameters, scaling factors, apply or remove stimulation or spatial constraints (e.g., local connectivity), or make any other change that does not alter the spatiotemporal domain of the system or its output (integration step, transmission speed and spatial support) and then resumed without the need of creating a new Simulator instance. Furthermore, this capability opens the possibility to dynamically update the simulation at runtime. Such a dynamic approach leads toward an adaptive modeling scheme where stimuli and other factors may be regulated by the ongoing activity (this last feature can be handled only from the scripting interface for the moment).

\section{DISCUSSION}

We have presented the architecture and usage of TVB, a neuroinformatics platform developed for simulations of network models of the full brain. Its scientific core has been developed by integrating concepts from theoretical, computational, cognitive and clinical neuroscience, with the aim to integrate neuroimage modalities along with the interacting mesoscopic and macroscopic scales of a biophysical model of the brain. From a computational modeling perspective TVB constitutes an alternative to approaches such as the work of Riera et al. (2005) and more recently that of Valdes-Sosa et al. (2009), as well as other relevant studies mentioned in the main text of this article. From a neuroinformatics perspective, TVB lays the groundwork for the integration of existing paradigms in the theory of large-scale models of the brain, by providing a general and flexible framework where the advantages and limitations of each approach may be determined. It also provides the community with a technology, that until now had not been publicly available, accessible by researchers with different levels and backgrounds, enabling systematic implementation and comparison of neural mass and neural field models, incorporating biologically realistic connectivity and cortical geometry and with the potential to become a novel tool for clinical interventions. While many other environments simulate neural activity at the level of neurons (Brian simulator, MOOSE, PCSIM, NEURON, NEST, GENESIS) (Hines and Carnevale, 2001; Gewaltig and Diesmann, 2007; Goodman and Brette, 2008; Ray and Bhalla, 2008; Pecevski et al., 2009; Brette and Goodman, 2011), even mimicking a number of specific brain functions (Eliasmith et al., 2012), they, most importantly, do not consider the space-time structure of full brain connectivity constraining whole brain neurodynamics, as a crucial component in their modeling paradigm. Other approaches to multi-modal integration such as Statistical Parametric Mapping (SPM) perform statistical fitting to experimental data at the level of a small set of nodes (Friston et al., 1995, 2003; David et al., 2006; Pinotsis and Friston, 2011) [i.e., they are data-driven as in Freestone et al. (2011)], thus diverging from our approach that could be categorized as a purely "computational neural modeling" paradigm as described in Bojak et al. (2011). From this perspective, the goal is to capture and reproduce whole brain dynamics by building a network constrained by its structural large-scale connectivity and mesoscopic models governing the nodes intrinsic dynamics.

Also, the extension of neuronal level modeling to large brain structures requires vast supercomputers to emulate the large 

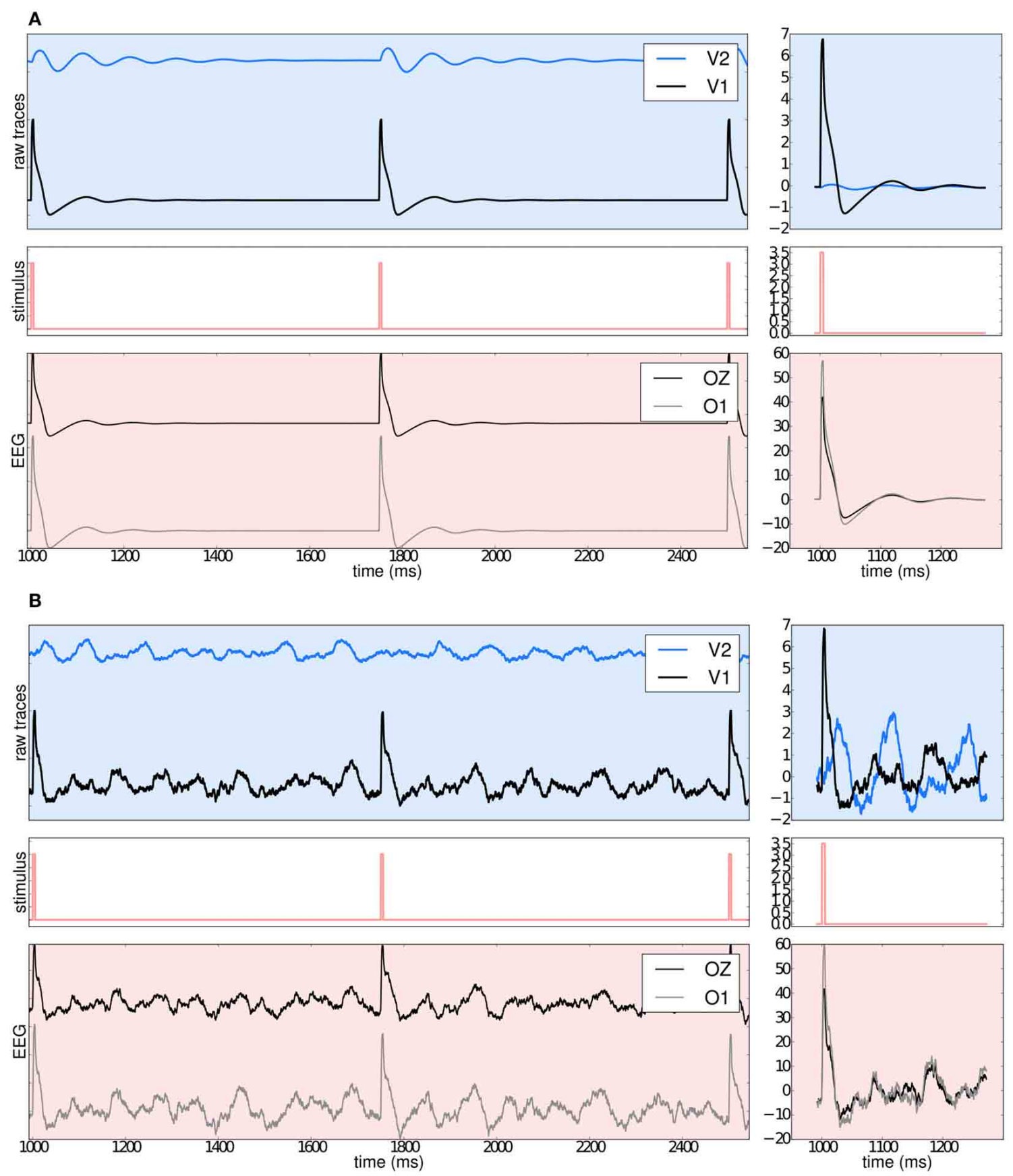

FIGURE 11 | (A) The upper left blue panel shows the raw traces of nodes V2 and $\mathrm{V} 1$; the latter stimulated with a rectangular pulse of width equal to $5 \mathrm{~ms}$ and repetition frequency of $1 \mathrm{~Hz}$. Signals are normalized by their corresponding maximum value. The right blue panel show the signals for a shorter period of time. Amplitudes are not normalized to emphasize the relative difference between the two regions. Middle panels illustrate the stimulus pattern. Lower red panels display the activity as projected onto EEG space and recorded from channels $\mathrm{Oz}$ and $\mathrm{O} 1$. The default EEG cap in TVB consists of 62 scalp electrodes distributed according to the 10-20 international system (Klem et al., 1999). In this simulation a deterministic integration scheme was employed to obtain the time-series of neural activity, since noise was not applied to the model's equations. (B) The same description as in (A) applies. The main difference with the previous simulation is that here white noise was added to the system. number of complex functional units. Focusing on the brain's large-scale architecture, in addition to the dimension reduction accomplished through the mean field methods applied on the mesoscopic scale, TVB allows for computer simulations on the full brain scale on workstations and small computing clusters, with no need to use supercomputing resources.

The simulator component of TVB has the goal of simulating mesoscopic neural dynamics on large-scale brain networks. 

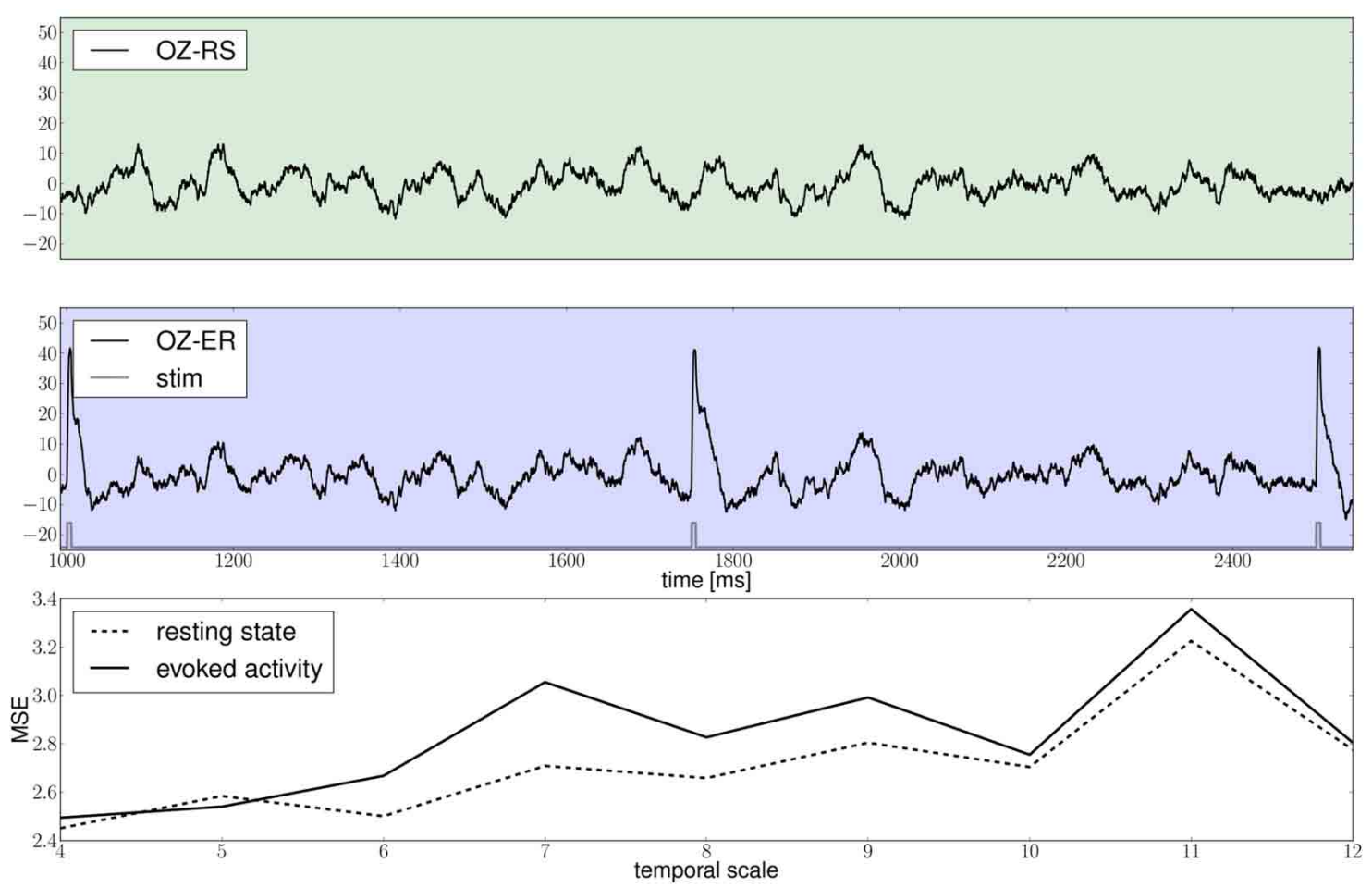

FIGURE 12 | The green and blue panels show EEG recordings from electrode $\mathrm{Oz}$ during the resting state, i.e., in the absence of stimulation and in the stimulated condition, respectively, notice the slow damped oscillations after stimulus onset at a approximately $10 \mathrm{~Hz}$; the light gray trace depicts the stimulation pattern. The bottom panel displays multiscale entropy estimates computed on the $\mathrm{Oz}$ time-series at different temporal scales using the dataset obtained by means of a stochastic integration scheme.
It does not intend to build brain models at the level of neurons (Goodman and Brette, 2009; Cornelis et al., 2012), however, it does leverage information from microscopic models to add detail and enhance the performance of the neural population models, which act as building blocks and functional units of the network. TVB thus represents a unique tool to systematically investigate the dynamics of the brain, emphasizing its large-scale network nature and moving away from the study of isolated regional responses, thereby considering the function of each region in terms of the interplay among brain regions. The primary spatial support (neuroanatomical data) on top of which the large-scale network model is built has a number of implications:

1. It constraints the type of network dynamics; dynamics that could be further related to physiology and behavior (Senden et al., 2012).

2. It permits a systematic investigation of the consequences of the particular restrictions imposed by that large-scale structure and the effect of changes to it.

3. It provides a reliable and geometrically accurate model of sources of neural activity, enabling realistic forward solutions to EEG/MEG based on implementations of boundary element methods (BEM) or other approaches such as finite difference time domain methods (FDTD).
On the basis of the literature, theoretical and clinical studies seeking to better understand and describe certain brain functions and structure use stimulation as an essential part of their protocols. Stimulation is a way to probe how the system respond under external perturbations adapting itself to the new environmental conditions or to categorize responses when stimulation represents real-life (visual, auditory, motor) sensory inputs. Among the current features of TVB, the easy generation of a variety of stimulation patterns is to be recognized as one of its great advantages and contributions to experimental protocol design. TVB permits the development of simple stimulation routines, allowing evaluation of the viability and usefulness of certain stimulation procedures.

TVB represents a powerful research platform, combining experimental design and numerical simulations into a collaborative framework that allows sharing of results and the integration of data from other applications. Naturally, this leads to the potential for an increased level of interaction among researchers of the broad neuroscience community. In the same direction, TVB is also an extensible validation platform since it supports the creation of basic modeling refinement loops, making model exploration and validation a relatively automated procedure. For instance, after generating a brain network model, exploring the system's parameter space by adjusting parameters of both the local dynamics and the large scale structure can be achieved with ease. Further, effects of local dynamics and network structure 
can be disentangled by evaluating distinct local dynamic models on the same structure or the same local dynamic model coupled through distinct structures. This constrained flexibility makes it easy for modelers to test new approaches, directly compare them with existing approaches and reproduce their own and other researchers' results. Reproducibility is indeed a required feature to validate and consequently increase the reliability of scientific work (Donoho, 2010) and the extensibility of TVB's scientific components, granted by its modular design, provides a mechanism to help researchers achieve this.

The brain network models of TVB, being built on explicit anatomical structure, enable modeling investigations of practical clinical interest. Specifically, whenever a dysfunction or disease expresses itself as a change to the large scale network structure, for instance, in the case of lesions in white-matter pathways, the direct replication of this structural change in TVB's brain network models is straight forward.

\section{FUTURE WORK}

Regarding performance, of special importance will be to evaluate all the parameters that have an effect on both memory usage and execution time for surface-based simulations. The reason is that realistic brain network models are built on top of surface meshes constructed by thousands of vertices per hemisphere $\left(2^{13}\right.$ for the TVB demonstration cortical surface) but can easily have more than 40,000 .

Equally important is to develop more tests to generally evaluate the simulation engine, paying close attention to keep the consistency and stability of the algorithms currently implemented.

Another aspect that deserves careful attention is the description of our modeling approach that was largely beyond the scope of this text. Therefore, the theory underlying the different methods involved in the development of a generalized framework

\section{REFERENCES}

Amari, S. (1975). Homogeneous nets of neuron-like elements. Biol. Cybern. 17, 211-220. doi: 10.1007/ bf00339367

Amari, S. (1977). Dynamics of pattern formation in lateral-inhibition type neural fields. Biol. Cybern. 22, 77-87. doi: 10.1007/BF00337259

Assisi, C., Jirsa, V., and Kelso, J. (2005). Synchrony and clustering in heterogeneous networks with global coupling and parameter dispersion. Phys. Rev. Lett. 94:018106. doi: 10.1103/PhysRevLett.94.018106

Atay, F., and Hutt, A. (2006). Neural fields with distributed transmission speeds and long range feedback delays. SIAD 5, 670-698. doi: 10.1137/050629367

Babajani-Feremi, A., and SoltanianZadeh, H. (2010). Multi-area neural mass modeling of eeg and meg signals. Neuroimage 52, 793-811. doi: 10.1016/ j.neuroimage.2010.01.034
Bakker, R., Wachtler, T., and Diesmann, M. (2012). Cocomac 2.0 and the future of tract-tracing databases. Front. Neuroinform. 6:30. doi: 10.3389/fninf.2012.00030

Bastiani, M., Shah, N. J., Goebel, R., and Roebroeck, A. (2012). Human cortical connectome reconstruction from diffusion weighted mri: the effect of tractography algorithm. Neuroimage 62, 1732-1749. doi: 10.1016/j.neuroimage.2012.06.002

Beurle, R. L. (1956). Properties of a mass of cells capable of regenerating pulses. Philos. Trans. R. Soc. Lond. B Biol. Sci. 240, 55-94. doi: 10.1098/rstb.1956.0012

Bojak, I., and Liley, D. T. J. (2010). Axonal velocity distributions in neural field equations. PLoS Comput. Biol. 6:e1000653. doi: 10.1371/journal.pcbi.1000653

Bojak, I., Oostendorp, T., Reid, A., and Kötter, R. (2011). Towards a modelbased integration of co-registered electroencephalography/functional

for brain network models is to be presented in future scientific publications.

To allow a most optimal dissemination of knowledge in TVB we are currently developing a web-based educational platform that will allow training on the usage of TVB, as well as serve as a key reference.

As simulations in TVB are built on the large-scale anatomical structure of the human brain, continued work to integrate new, reliable, sources of structural data is essential to the progress of the platform. An obvious future resource in this regard will be the newly developed database of the Human Connectome Project (Essen and Ugurbil, 2012; Essen et al., 2012).

\section{INFORMATION SHARING STATEMENT (LICENSE)}

The data and software in this study belong to an ongoing project; it is free software and licensed under the GNU General Public License version 2 as published by the Free Software Foundation. The latest releases of The Virtual Brain including the source code and demo data are free to download from http://www. thevirtualbrain.org. The source code available in the public repository includes the latest experimental features regarding GPU implementation.

\section{ACKNOWLEDGMENTS}

Funding: The research reported herein was supported by the Brain Network Recovery Group through the James S. McDonnell Foundation and the FP7-ICT BrainScales. Paula Sanz Leon is supported by a doctoral fellowship from Ministere de la Recherche.

\section{SUPPLEMENTARY MATERIAL}

The Supplementary Material for this article can be found online at: http://www.frontiersin.org/Neuroinformatics/10.3389/fninf. 2013.00010/abstract

magnetic resonance imaging data with realistic neural population meshes. Philos. Trans. $R$ Soc. Lond. A. 369, 3785-3801. doi: 10.1098/rsta.2011.0080

Bojak, I., Oostendorp, T., Reid, A., and R, K. (2010). Connecting mean field models of neural activity to eeg and fmri data. Brain Topogr. 23, 139-149. doi: 10.1007/s10548-0100140-3

Bostock, M., Ogievetsky, V., and Heer, J. (2011). D3 data-driven documents. IEEE Trans. Visual. Comput. Graphics 17, 2301-2309. doi: 10.1109/TVCG.2011.185

Breakspear, M., and Jirsa, V. (2007). Handbook of Brain Connectivity (Understanding Complex Systems) Neuronal Dynamics and Brain Connectivity. Berlin; Heidelberg: Springer.

Breakspear, M., Roberts, J. A., Terry, J. R., Rodrigues, S., Mahant, N., and Robinson, P. A. (2006). A unifying explanation of primary generalized seizures through nonlinear brain modeling and bifurcation analysis. Cereb. Cortex 16, 1296-1313. doi: 10.1093/cercor/ bhj072

Breakspear, M., Terry, J. R., and Friston, K. J. (2003). Modulation of excitatory synaptic coupling facilitates synchronization and complex dynamics in a biophysical model of neuronal dynamics. Network 14, 703-732. doi: 10.1088/0954-898X/14/4/305

Bressloff, P. C. (2012). From invasion to extinction in heterogeneous neural fields. JMN 2:6. doi: 10.1186/21908567-2-6

Brette, R., and Goodman, D. F. M. (2011). Vectorized algorithms for spiking neural network simulation. Neural Comput. 23, 1503-1535. doi: 10.1162/NECO_a_00123

Brunel, N., and Wang, X.-J. (2001). Effects of neuromodulation in a cortical network model of object working memory dominated by 
recurrent inhibition. J. Comput. Neurosci. 11, 63-85.

Brunel, N., and Wang, X.-J. (2003). What determines the frequency of fast network oscillations with irregular neural discharges? i. synaptic dynamics and excitation-inhibition balance. J. Neurophysiol. 90, 415-430. doi: 10.1152/jn.01095. 2002

Bullmore, E., and Sporns, O. (2009). Complex brain networks: graph theoretical analysis of structural and functional systems. Nat. Rev. Neurosci. 10, 186-198. doi: 10.1038/ nrn2575

Burrage, K., Burrage, P. M., and Tian, T. (2004). Numerical methods for strong solutions of stochastic differential equations: an overview. Proc. R. Soc. Lond. A 460, 373-402. doi: 10.1098/rspa.2003.1247

Buxton, R., and Frank, L. (1997). A model for the coupling between cerebral blood flow and oxygen metabolism during neural stimulation. J. Cereb. Blood Flow Metab. 17, 64-72. doi: 10.1097/00004647199701000-00009

Buzsaki, G. (2006). Rhythms of the Brain. Oxford: Oxford University Press.

Chacon, S. (2009). Pro Git. Berkeley, CA: Apress.

Coombes, S. (2010). Large-scale neural dynamics: simple and complex. Neuroimage 52, 731-739. doi: 10.1016/j.neuroimage.2010.01.045

Cornelis, H., Rodriguez, A. L., Coop, A. D., and Bower, J. M. (2012). Python as a federation tool for genesis 3.0. PLoS ONE 7:e29018. doi: 10.1371/journal.pone.0029018

Costa, M., Goldberger, A. L., and Peng, C.-K. (2002). Multiscale entropy analysis of complex physiologic time series. Phys. Rev. Lett. 89:068102. doi: 10.1103/PhysRevLett.89.068102

Costa, M., Goldberger, A. L., and Peng, C.-K. (2005). Multiscale entropy analysis of biological signals. Phys. Rev. E Stat. Nonlin. Soft Matter Phys. 71(2 Pt 1):021906. doi: 10.1103/ PhysRevE.71.021906

David, O., Kilner, J. M., and Friston, K. J. (2006). Mechanisms of evoked and induced responses in MEG/EEG. Neuroimage 31, 1580-1591. doi: 10.1016/ j.neuroimage.2006.02.034

Deco, G., and Jirsa, V. (2012). Ongoing cortical activity at rest: criticality, multistability, and ghost attractors. J. Neurosci. 32, 3366-3375. doi: 10.1523/JNEUROSCI.2523-11.2012

Deco, G., Jirsa, V., and McIntosh, A. (2011). Emerging concepts for the dynamical organization of resting-state activity in the brain. Nat. Rev. Neurosci. 12, 43-56. doi: 10.1038/nrn2961

Deco, G., Jirsa, V., McIntosh, A. Sporns, O., and Kötter, R. (2009). Key role of coupling, delay, and noise in resting brain fluctuations. Proc. Natl. Acad. Sci. U.S.A. 106, 10302-10307. doi: 10.1073/ pnas.0901831106

Deco, G., Jirsa, V., Robinson, P. A., Breakspear, M., and Friston, K. (2008). The dynamic brain: from spiking neurons to neural masses and cortical fields. PLoS Comput. Biol. 4:e1000092. doi: 10.1371/ journal.pcbi.1000092

Donoho, D. L. (2010). An invitation to reproducible computational research. Biostatistics 11, 385-388. doi: 10.1093/biostatistics/kxq028

Eliasmith, C., Stewart, T. C., Choo, X., Bekolay, T., DeWolf, T., Tang, Y., et al. (2012). A large-scale model of the functioning brain. Science $338,1202-1205$. doi: 10.1126/ science. 1225266

Enthought, I. (2001). The traits framework for validation and event-driven programming in python. Available online at: http://code.enthought.com/projects/ traits/

Essen, D. C. V., and Ugurbil, K. (2012). The future of the human connectome. Neuroimage 62, 1299-1310. doi: 10.1016/ j.neuroimage.2012.01.032

Essen, D. C. V., Ugurbil, K., Auerbach, E., Barch, D., Behrens, T. E. J., Bucholz, R., et al. (2012). The human connectome project: a data acquisition perspective. Neuroimage 62, 2222-2231. doi: 10.1016/ j.neuroimage.2012.02.018

FitzHugh, R. (1961). Impulses and physiological states in theoretical models of nerve membrane. Biophys. J. 1, 445-466. doi: 10.1016/ S0006-3495(61)86902-6

Fox, R., Gatland, I., Rot, R., and Vemuri, G. (1988). Fast, accurate algorithm for numerical simulation of exponentially correlated colored noise. Phys. Rev. A 38, 5938-5940. doi: 10.1103/PhysRevA. 38.5938

Freeman, W. J. (1975). Mass Action in the Nervous System. New York; San Francisco; London: Academic press.

Freeman, W. J. (1992). Tutorial on neurobiology: from single neurons to brain chaos. Int. J. Bif. Chaos 2, 451-482. doi: 10.1142/ S0218127492000653

Freestone, D. R., Aram, P., Dewar, M., Scerri, K., Grayden, D. B. and Kadirkamanathan, V. (2011). A data-driven framework for neural field modeling. Neuroimage 56, 1043-1058. doi: 10.1016/ j.neuroimage.2011.02.027

Freyer, F., Reinacher, M., Nolte, G. Dinse, H. R., and Ritter, P. (2012) Repetitive tactile stimulation changes resting-state functional connectivity-implications for treatment of sensorimotor decline. Front. Hum. Neurosci. 6:144. doi 10.3389/fnhum.2012.00144

Freyer, F., Roberts, J. A., Becker, R., Robinson, P. A., Ritter, P., and Breakspear, M. (2011). Biophysical mechanisms of multistability in resting-state cortical rhythms. J. Neurosci. 31, 6353-6361. doi: 10.1523/JNEUROSCI.6693-10.2011

Friston, K., Harrison, L., and Penny, W. (2003). Dynamic causal modelling. Neuroimage 19, 1273-1302. doi: 10.1016/ S1053-8119(03)00202-7

Friston, K., Holmes, A., Worsley, K. Poline, J., Frith, C., and Frackowiak R. (1995). Statistical parametric maps in functional imaging: a general linear approach. Hum. Brain Mapp. 2, 189-210. doi: 10.1002/ hbm.460020402

Friston, K. J., Mechelli, A., Turner R., and Price, C. J. (2000) Nonlinear responses in fMRI the balloon model, volterra kernels, and other hemodynamics. Neuroimage 12, 466-477. doi: 10.1006/nimg.2000.0630

Gerhard, S., Daducci, A., Lemkaddem, A., Meuli, R., Thiran, J.-P., and Hagmann, P. (2011). The connectome viewer toolkit: an open source framework to manage, analyze, and visualize connectomes. Front. Neuroinform. 5:3. doi: 10.3389/ fninf.2011.00003

Gewaltig, M., and Diesmann, M. (2007). NEST (neural simulation tool). Scholarpedia 2:1430. doi: 10.4249/scholarpedia.1430

Ghosh, A., Rho, Y., McIntosh, A., Kötter, R., and Jirsa, V. (2008). Noise during rest enables the exploration of the brain's dynamic repertoire. PLoS Comput. Biol. 4:e1000196. doi: 10.1371/journal.pcbi.1000196

Goodale, M. A., and Milner, A. D. (1992). Separate visual pathways for perception and action. Trends Neurosci. 15, 20-25. doi: 10.1016/0166-2236(92)90344-8

Goodman, D. F. M., and Brette, R. (2008). Brian: a simulator for spiking neural networks in python. Front. Neuroinform. 2:5. doi: 10.3389/neuro.11.005.2008

Goodman, D. F. M., and Brette, R. (2009). The brian simulator Front. Neurosc. 3, 192-197. doi: 10.3389/neuro.01.026.2009
Gramfort, A., Papadopoulo, T. Olivi, E., and Clerc, M. (2010). Openmeeg: opensource software for quasistatic bioelectromagnetics. Biomed. Eng. Online 9:45. doi: 10.1186/1475-925X-9-45

Hagmann, P., Cammoun, L., Gigandet, X., Meuli, R., Honey, C. J., Wedeen, V. J., et al. (2008). Mapping the structural core of human cerebral cortex. PLoS Biol. 6:e159. doi: 10.1371/journal. pbio.0060159

Haken, H. (1983). Synergetics, an Introduction: Nonequilibrium Phase Transitions and Self-Organization in Physics, Chemistry, and Biology. 3rd Edn. New York, NY: Springer Verlag.

Haken, H. (2001). Delay, noise and phase locking in pulse coupled neural networks. Biosystems 63, 15-20. doi: 10.1016/S0303-2647 (01)00143-5

Hindmarsh, J., and Rose, R. (1984). A model of neuronal bursting using three coupled first order differential equations. Proc. $R$. Soc. Lond. Ser. B 221, 87-122. doi: 10.1098/rspb.1984.0024

Hinds, O., Polimeni, J. R., Rajendran, N., Balasubramanian, M., Amunts, K., Zilles, K., et al. (2009). Locating the functional and anatomical boundaries of human primary visual cortex. Neuroimage 46, 915-922. doi: 10.1016/ j.neuroimage.2009.03.036

Hines, M. L., and Carnevale, N. T. (2001). Neuron: a tool for neuroscientists. Neuroscientist 7, 123-135.

Hämäläinen, M. S. (1992). Magnetoencephalography: a tool for functional brain imaging. Brain Topogr. 5, 95-102.

Hämäläinen, M. S., Hari, R., Ilmoniemi, R. J., Knuutila, J., and Lounasmaa, O. V. (1993). Magnetoencephalography-theory, instrumentation, and applications to noninvasive studies of the working human brain. Rev. Modern Phys. 65, 413-497. doi: 10.1103/ revmodphys.65.413

Honey, C. J., Sporns, O., Cammoun, L., Gigandet, X., Thiran, J. P., Meuli, R., et al. (2009). Predicting human resting-state functional connectivity from structural connectivity. Proc. Natl. Acad. Sci. U.S.A. 106, 2035-2040. doi: $10.1073 /$ pnas.0811168106

Hunter, J. D. (2007). Matplotlib: a 2d graphics environment. Comput. Sci. Eng. 9, 90-95. doi: 10.1109/ mcse. 2007.55

Jansen, B., and Rit, V. (1995) Electroencephalogram and visual evoked potential generation in a 
mathematical model of coupled cortical columns. Biol. Cybern. 73, 357-366. doi: 10.1007/BF00199471

Jansen, B., Zouridakis, G., and Brandt, M. (1993). A neurophysiologicallybased mathematical model of flash visual evoked potentials. Biol. Cybern. 68, 275-283. doi: 10.1007/ BF00224863

Jirsa, V. (2004). Connectivity and dynamics of neural information processing. Neuroinformatics 2, 183-204. doi: 10.1385/NI:2:2:183

Jirsa, V., and Haken, H. (1996). Field theory of electromagnetic brain activity. Phys. Rev. Lett. 77, 960-963. doi: 10.1103/PhysRevLett.77.960

Jirsa, V., and Haken, H. (1997). A derivation of a macroscopic field theory of the brain from the quasimicroscopic neural dynamics. Phys. D 99, 503-526. doi: 10.1016/S01672789(96)00166-2

Jirsa, V., and Kelso, J. A. (2000). Spatiotemporal pattern formation in neural systems with heterogeneous connection topologies. Phys. Rev. E Stat. Phys. Plasmas Fluids Relat. Interdisciplin. Topics $62(6$ Pt B), 8462-8465. doi: 10.1103/ PhysRevE.62.8462

Jirsa, V., Jantzen, K., Fuchs, A., and Kelso, J. (2002). Spatiotemporal forward solution of the eeg and meg using network modeling. IEEE Trans. Med. Imag. 21, 493-504. doi: 10.1109/TMI.2002.1009385

Jirsa, V., and Stefanescu, R. (2010). Neural population modes capture biologically realistic large scale network dynamics. Bull. Math. Biol. 73, 325-343. doi: 10.1007/s11538-0109573-9

Jirsa, V., Sporns, O., Breakspear, M., Deco, G., and McIntosh, A. R. (2010). Towards the virtual brain: network modeling of the intact and the damaged brain. Arch. Ital. Biol. 148, 189-205.

Kelso, S. (1995). Dynamic Patterns: The Self-Organization of Brain and Behavior (Complex Adaptive Systems). Cambridge, MA: MIT Press.

Klöden and Platen (1995). Numerical Solution of Stochastic Differential Equations. Berlin: Springer.

Klem, G. H., Lüders, H. O., Jasper, H. H., and Elger, C. (1999). The ten-twenty electrode system of the international federation. the international federation of clinical neurophysiology. Electroencephalogr. Clin. Neurophysiol. Suppl. 52, 3-6.

Knock, S., McIntosh, A., Sporns, O., Kötter, R., Hagmann, P., and Jirsa, V. (2009). The effects of physiologically plausible connectivity structure on local and global dynamics in large scale brain models. J. Neurosci. Methods 183 86-94. doi: 10.1016/j.jneumeth. 2009.07.007

Kötter, R. (2004). Online retrieval, processing, and visualization of primate connectivity data from the cocomac database. Neuroinformatics 2, 127-144. doi: 10.1385/NI:2:2:127

Kötter, R., and Wanke, E. (2005). Mapping brains without coordinates. Philos. Trans. R. Soc. Lond. $B$ Biol. Sci. 360, 751-766. doi: 10.1098/rstb.2005.1625

Lake, D. E., and Moorman, J. R. (2011). Accurate estimation of entropy in very short physiological time series: the problem of atrial fibrillation detection in implanted ventricular devices. Am. J. Physiol. Heart Circ. Physiol. 300, H319-H325. doi: 10.1152/ajpheart.00561.2010

Liley, D. T. J., and Bojak, I. (2005). Understanding the transition to seizure by modeling the epileptiform activity of general anesthetic agents. J. Clin. Neurophysiol. 22, 300-313.

Logothetis, N. K., Pauls, J., Augath, M., Trinath, T., and Oeltermann, A. (2001). Neurophysiological investigation of the basis of the fmri signal. Nature 412, 150-157. doi: $10.1038 / 35084005$

Lopes da Silva, F. H., Hoeks, A., Smits, H., and Zetterberg, L. H. (1974). Model of brain rhythmic activity. Biol. Cybern. 15, 27-37. doi: 10.1007/BF00270757

McIntosh, A., Kovacevic, N., Lippe, S., Garrett, D., Grady, C., and Jirsa, V. (2010). The development of a noisy brain. Arch. Ital. Biol. 148, 323-337.

Mosher, J., Leahy, R., and Lewis, P. (1999). EEG and MEG: forward solutions for inverse methods. IEEE Trans. Biomed. Eng. 46, 245-259.

Nagumo, J. (1962). An active pulse transmission line simulating nerve axon. Proc. IRE. 50, 2061-2070. doi: 10.1109/jrproc. 1962.288235

Niedermeyer, E., and Lopes Da Silva, F., H., (eds.). (2005). Electroencephalography: Basic Principles, Clinical Applications, and Related Fields. Philadelphia, PA: Lippincott Williams \& Wilkins.

Nolte, G., Bai, O., Wheaton, L., Mari, Z., Vorbach, S., and Hallett, M. (2004). Identifying true brain interaction from eeg data using the imaginary part of coherency. Clin. Neurophysiol. 115, 2292-2307. doi: 10.1016/j.clinph.2004.04.029
Nunez, P. (1974). The brain wave equation: a model for the EEG. Math. Biosci. 21, 279-297. doi 10.1016/0025-5564(74)90020-0

Nunez, P., L., (ed.). (1995). Neocortical Dynamics and Human EEG Rhythms. New York, NY: Oxford University Press.

Nunez, P., L., and Srinivasan, R., (eds.) (1981). Electric Fields of the Brain: The Neurophysics of EEG. New York, NY: Oxford University Press.

Ogawa, S., Menon, R. S., Kim, S. G., and Ugurbil, K. (1998). On the characteristics of functional magnetic resonance imaging of the brain. Annu. Rev. Biophys. Biomol. Struct. 27, 447-474. doi: 10.1146/annurev.biophys.27.1.447

Ogawa, S., Menon, R. S., Tank, D. W., Kim, S. G., Merkle, H., Ellermann, J. M., et al. (1993). Functional brain mapping by blood oxygenation level-dependent contrast magnetic resonance imaging. a comparison of signal characteristics with a biophysical model. Biophys. J. 64, 803-812. doi: 10.1016/S0006-3495 (93)81441-3

Oliphant, T. E. (2006). Guide to NumPy. Trelgol Publishing.

Pecevski, D., Natschläger, T., and Schuch, K. (2009). Pcsim: a parallel simulation environment for neural circuits fully integrated with python. Front. Neuroinform. 3:11. doi: 10.3389/neuro.11.011.2009

Pedregosa, F., Varoquaux, G., Gramfort, A., Michel, V., Thirion, B., Grisel, O., et al. (2011). Scikitlearn: machine learning in Python JMLR 12, 2825-2830.

Pinotsis, D. A., and Friston, K. J. (2011). Neural fields, spectral responses and lateral connections. Neuroimage 55, 39-48. doi: 10.1016/j.neuroimage.2010.11.081

Pinotsis, D. A., Moran, R. J., and Friston, K. J. (2012). Dynamic causal modeling with neural fields. Neuroimage 59, 1261-1274. doi: 10.1016/j.neuro image.2011.08.020

Ramachandran, P., and Varoquaux, G. (2011). Mayavi: 3D visualization of scientific data. Comput. Sci. Eng. 13, 40-51. doi: 10.1109/mcse.2011.35

Ray, S., and Bhalla, U. S. (2008). Pymoose: interoperable scripting in python for moose. Front. Neuroinform. 2:6. doi: 10.3389/ neuro.11.006.2008

Rennie, C. J., Robinson, P. A., and Wright, J. J. (1999). Effects of local feedback on dispersion of electrical waves in the cerebral cortex. Phys. Rev. E 59, 3320-3329. doi: 10.1103/PhysRevE.59.3320
Rennie, C. J., Robinson, P. A., and Wright, J. J. (2002). Unified neurophysical model of eeg spectra and evoked potentials. Biol. Cybern. 86, 457-471. doi: 10.1007/s00422-0020310-9

Riera, J., Aubert, E., Iwata, K., Kawashima, R., Wan, X., and Ozaki, T. (2005). Fusing eeg and fmri based on a bottom-up model: inferring activation and effective connectivity in neural masses. Philos. Trans. R. Soc. Lond. B. Biol. Sci. 360, 1025-1041. doi: 10.1098/ rstb.2005.1646

Ritter, P., Schirner, M., McIntosh, A., and VK, J. (2013). The virtual brain integrates computational modelling and multimodal neuroimaging. Brain Connect. 3, 121-145. doi: 10.1089/brain.2012.0120

Robinson, P., Rennie, C., and Wright, J. (1997). Propagation and stability of waves of electrical activity in the cerebral cortex. Phys. Rev. E 56, 826-840. doi: 10.1103/PhysRevE.56.826

Robinson, P. A. (2011). Neural field theory of synaptic plasticity. $J$. Theor. Biol. 285, 156-163. doi: 10.1016/j.jtbi.2011.06.023

Robinson, P. A., Rennie, C. J., Rowe, D. L., O'Connor, S. C., Wright, J. J., Gordon, E., et al. (2003). Neurophysical modeling of brain dynamics. Neuropsychopharmacology 28 (Suppl. 1), S74-S79. doi: 10.1038/ sj.npp. 1300143

Robinson, P. A., Rennie, C. J., Wright, J. J., Bahramali, H., Gordon, E., and Rowe, D. L. (2001). Prediction of electroencephalographic spectra from neurophysiology. Phys. Rev. E 63(2 Pt 1):021903. doi: 10.1103/ PhysRevE.63.021903

Rowe, D. L., Robinson, P. A., and Rennie, C. J. (2004). Estimation of neurophysiological parameters from the waking EEG using a biophysical model of brain dynamics. J. Theor. Biol. 231, 413-433. doi: 10.1016/j.jtbi.2004.07.004

Rubinov, M., and Sporns, O. (2010). Complex network measures of brain connectivity: uses and interpretations. Neuroimage 52, 1059-1069. doi: 10.1016/j.neuro image.2009.10.003

Sarvas, J. (1987). Basic mathematical and electromagnetic concepts of the biomagnetic inverse problems. Phys. Med. Biol. 32, 11-22. doi: 10.1088/0031-9155/ $32 / 1 / 004$

Schneider, G. E. (1969). Two visual systems. Science 163, 895-902. doi: $10.1126 /$ science. 163.3870 .895 
Senden, M., Goebel, R., and Deco, G. (2012). Structural connectivity allows for multi-threading during rest: the structure of the cortex leads to efficient alternation between resting state exploratory behavior and default mode processing. Neuroimage 60, 2274-2284. doi: 10.1016/ j.neuroimage.2012.02.061

Shreiner, D., Woo, M., Neider, J. and Davis, T. (2005). Open $G L(R)$ Programming Guide: The Official Guide to Learning OpenGL(R). Version 2, 5th Edn. Addison-Wesley Professional. Available online at: http://www.glprogramming.com/ red/about.html

Sotero, R. C., and Trujillo-Barreto, N. J. (2008). Biophysical model for integrating neuronal activity, EEG, fMRI and metabolism. Neuroimage 39, 290-309. doi: 10.1016/j.neuroimage.2007.08.001

Sotero, R. C., Trujillo-Barreto, N. J., Iturria-Medina, Y., Carbonell, F., and Jimenez, J. C. (2007). Realistically coupled neural mass models can generate eeg rhythms. Neural Comput. 19, 478-512. doi: 10.1162/neco.2007.19.2.478

Spacek, M., Blanche, T., and Swindale, N. (2008). Python for large-scale electrophysiology. Front. Neuroinform. 2:9. doi: 10.3389/neuro.11.009.2008

Spiegler, A., Kiebel, S. J., Atay, F. M., and Knösche, T. R. (2010). Bifurcation analysis of neural mass models: impact of extrinsic inputs and dendritic time constants. Neuroimage 52, 1041-1058. doi: 10.1016/ j.neuroimage.2009.12.081

Stefanescu, R., and Jirsa, V. (2008). A low dimensional description of globally coupled heterogeneous neural networks of excitatory and inhibitory. PLoS Comput. Biol. 4, 26-36. doi: 10.1371/ journal.pcbi.1000219

Stefanescu, R., and Jirsa, V. (2011). Reduced representations of heterogeneous mixed neural networks with synaptic coupling. Phys. Rev. E 83:026204. doi: 10.1103/PhysRevE.83.026204

The HDF Group. (2000-2010). Hierarchical data format version 5. Available online at: http://www. hdfgroup.org/

Valdes-Sosa, P. A., Sanchez-Bornot, J. M., Sotero, R. C., IturriaMedina, Y., Aleman-Gomez, Y., Bosch-Bayard, J., et al. (2009). Model driven EEG/fMRI fusion of brain oscillations. Hum. Brain Mapp. 30, 2701-2721. doi: 10.1002/hbm.20704

von Ellenrieder, N., Beltrachini, L., and Muravchik, C. H. (2012). Electrode and brain modeling in stereo-EEG. Clin. Neurophysiol. 123, 1745-1754 doi: 10.1016/j.clinph.2012.01.019

Wilson, H., and Cowan, J. (1972) Excitatory and inhibitory interactions in localized populations of model neurons. Biophys. J. 12, 1-24. doi: 10.1016/S00063495(72)86068-5

Wilson, H., and Cowan, J. (1973). A mathematical theory of the functional dynamics of cortical and thalamic nervous tissue. Kybernetik 13 , 55-80. doi: 10.1007/bf00288786

Wong, K.-F., and Wang, X.-J. (2006) A recurrent network mechanism of time integration in perceptual decisions. J. Neurosci. 26, 1314-1328. doi: 10.1523/JNEUROSCI.3733-05. 2006

Wright, J. J., and Liley, D. T. J. (1995). Simulation of electrocortical waves. Biol. Cybern. 72, 347-356. doi: 10.1007/BF00202790

Zalesky, A., Fornito, A., Harding, I. H., Cocchi, L., Yücel, M. Pantelis, C., et al. (2010). Wholebrain anatomical networks: does the choice of nodes matter? Neuroimage 50, 970-983. doi: 10.1016/j.neuroimage.2009.12.027

Conflict of Interest Statement: The authors declare that the research was conducted in the absence of any commercial or financial relationships that could be construed as a potential conflict of interest.

Received: 01 March 2013; accepted: 22 May 2013; published online: 11 June 2013.

Citation: Sanz Leon $P$, Knock SA, Woodman MM, Domide L, Mersmann J, McIntosh AR and Jirsa V (2013) The Virtual Brain: a simulator of primate brain network dynamics. Front. Neuroinform. 7:10. doi: 10.3389/fninf.2013.00010 Copyright (c) 2013 Sanz Leon, Knock, Woodman, Domide, Mersmann, McIntosh and Jirsa. This is an open-access article distributed under the terms of the Creative Commons Attribution License, which permits use, distribution and reproduction in other forums, provided the original authors and source are credited and subject to any copyright notices concerning any third-party graphics etc. 